\title{
Model reduction of neutral linear and nonlinear time-invariant time-delay systems with discrete and distributed delays
}

\author{
Giordano Scarciotti and Alessandro Astolfi
}

\begin{abstract}
The problem of model reduction by moment matching for linear and nonlinear differential time-delay systems is studied. The class of models considered includes neutral differential time-delay systems with discrete-delays and distributeddelays. The description of moment is revisited by means of a Sylvester-like equation for linear time-delay systems and by means of the center manifold theory for nonlinear time-delay systems. In addition the moments at infinity are characterized for both linear and nonlinear time-delay systems. Parameterized families of models achieving moment matching are given. The parameters can be exploited to derive delay-free reduced order models or time-delay reduced order models with additional properties, e.g. interpolation at an arbitrary large number of points. Finally, the problem of obtaining a reduced order model of an unstable system is discussed and solved.
\end{abstract}

\section{INTRODUCTION}

$\mathbf{R}$ EDUCED order models are exploited in the prediction and control of a wide class of physical behaviors. For instance, reduced order models are used to simulate or design weather forecast models, very large scale integrated circuits or networked dynamical systems [1]. The model reduction problem consists in finding a simplified description of a dynamical system maintaining at the same time specific properties. It has been extensively studied exploiting the singular value decomposition (SVD), e.g. [2], [3], [4], [5], [6], [7], [8], and the Krylov projection method (also known as moment matching), e.g. [9], [10], [11], [12], [13], [14] and [15]. The advantage of the moment matching over the SVD-based methods is that the numerical implementation is much more efficient [1, Section 14.1]. The major drawback, however, is the difficulty in the moment matching methods to preserve properties of the original systems. In [16] and [17] a new interpretation of the moment matching problem has been given exploiting the center manifold theory. The outcome of these papers is the ability to maintain the properties of the original system and the possibility of extending the moment matching techniques to nonlinear systems. This has led to new results in the area of model reduction, see e.g. [18], [19], [20] and [21].

In this paper we extend the model reduction techniques based

G. Scarciotti is with the Dept. of Electrical and Electronic Engineering, Imperial College London, London, SW7 2AZ, UK, E-mail: gs3610@ic.ac.uk.

A. Astolfi is with the Dept. of Electrical and Electronic Engineering, Imperial College London, London, SW7 2AZ, UK and DICII, University of Rome "Tor Vergata", Via del Politecnico 1, 00133 Rome, Italy, E-mail: a.astolfi@ic.ac.uk. This work is partly supported by the EPSRC Programme Grant "Control For Energy and Sustainability" EP/G066477. on moment matching to a general class of linear and nonlinear differential time-delay systems. Time-delay systems are a class of infinite dimensional systems extensively studied in the literature, e.g. see the monographs [22], [23], [24], [25], [26], [27], [28] and the survey papers [29], [30] and [31]. From a practical point of view every dynamical system presents delays of some extent, see for instance [32] and [26], in which several examples from biology, chemistry, physics and engineering are discussed. Delays in closed-loop systems can generate unexpected behaviors (for example oscillations or instability). For instance "small" delays may be destabilizing [33], [34], [35], [30], [36] and [37], while "large" delays may be stabilizing [38], [39], [40] and [41].

The problem of model reduction of time-delay system is a classic topic in control theory. The optimal reduction (in the sense of some norm) is listed as an unsolved problem in systems theory in [42] and several results have been given using rational interpolations, e.g. [43], [44] and [45], see also [46], [47], [48], [49], [50], [51] and [52]. Recent results include model order reduction techniques for linear time-delay systems, see e.g. [53], [54], [55], and for infinite dimensional systems, see e.g. [56] and [57] in which operators are used to provide reduced order models for linear systems.

In this paper the model reduction method developed in [17] is extended to linear and nonlinear time-delay systems. For linear systems, it is shown that the moments of the system are fully characterized by the solution of a Sylvester-like equation. Although Sylvester equations have been widely studied (see for instance [58], [59]), some care is needed to extend the classical results to the particular Sylvester-like equation that arises in the paper. The results are then generalized to the class of linear neutral differential time-delay systems with discrete and distributed delays. A family of systems that achieve moment matching is characterized and connections with the results in [17] are drawn. As noted in [60] a reduced order model with time delays may lead to improvements in the approximation. Accordingly, the possibility to maintain the delay in the reduced order model is discussed and, in addition, it is shown that the introduction of delays can be used to improve the approximation, interpolating at a larger number of points.

For nonlinear systems, the description of moment is based on the center manifold theory for time-delay systems and is derived using the steady-state response of the system. For time-delay systems the conditions and properties of the center manifold hold as for finite dimensional systems (see e.g. [22], 
[61]). Exploiting this interpretation, a parameterized family of models described by differential time-delay equations is characterized. In addition, the notion of moment at infinity for linear and nonlinear time-delay systems is investigated and a brief discussion for more general types of nonlinear time-delay systems (i.e. neutral differential time-delay systems) is given. Finally, the problem of obtaining a reduced order model of the open-loop system given the closed-loop system is discussed and solved.

The rest of the paper is organized as follows. In Section II the notion of moment derived in [17] is extended to linear time-delay systems (Section II-A), the solution of the resulting Sylvester-like equation is discussed (Section II-B) and a revisitation of this description is given using the notion of steady-state response (Section II-C). The results are then extended to neutral systems and systems with distributeddelays (Section II-D). A family of systems achieving moment matching is presented (Sections II-E and II-F), the possibility of interpolating a larger number of points maintaining the same "number of equations" is investigated (Section II-G) and an example to illustrate the results is given. In Section III the center manifold theory for time-delay systems is used to extend the definition of moment to nonlinear time-delay systems (Section III-A) and a family of systems achieving moment matching for nonlinear time-delay systems is given (Sections III-B and III-C). The problem of "openloop" reduced order models is discussed (Section IV) and the characterization of the moments at infinity for linear and nonlinear time-delay systems is presented (Section V). Finally Section VI contains some concluding remarks.

Preliminary versions of this paper have been published in [62] and [63]. The original contribution of the present paper is that the class of systems is generalized including delays in the input and the output, the proofs of the theorems are given, the case of distributed-delays and neutral equations is considered, the possibility of exploiting the delays to interpolate at additional points is further investigated and an example is presented. Finally a class of "open-loop reduced order models" is given.

Notation. We use standard notation. $\mathbb{R}_{\geq 0}$ denotes the set of non-negative real numbers, $\mathbb{R}_{>0}$ denotes $\mathbb{R}_{\geq 0} \backslash\{0\}, \mathbb{C}_{<0}$ denotes the set of complex numbers with strictly negative real part and $\mathbb{D}_{<1}$ the set of complex numbers with absolute value less than one. Given a set of delays $\left\{\tau_{j}\right\}$, the symbol $\mathfrak{R}_{T}^{n}=\mathfrak{R}_{T}^{n}\left([-T, 0], \mathbb{R}^{n}\right)$, with $T=\max _{j}\left\{\tau_{j}\right\}$, indicates the set of continuous functions mapping the interval $[-T, 0]$ into $\mathbb{R}^{n}$ with the topology of uniform convergence. The symbol $I$ denotes the identity matrix, the symbol $\otimes$ indicates the Kronecker product and $\sigma(A)$ denotes the spectrum of the matrix $A \in \mathbb{R}^{n \times n} \cdot x_{\tau}$ denotes the translation operator that is $x_{\tau}(t)=x(t-\tau)$. Let $\bar{s} \in \mathbb{C}$ and $A(s) \in \mathbb{C}^{n \times n}$. Then $\bar{s} \notin \sigma(A(s))$ means that $\operatorname{det}(\bar{s} I-A(\bar{s})) \neq 0 . \sigma(A(s)) \subset \mathbb{C}_{<0}$ means that for all $s$ such that $\operatorname{det}(s I-A(s))=0, s \in \mathbb{C}_{<0}$. $\mathcal{L}(f(t))$ denotes the Laplace transform of the function $f(t)$ (provided that $f(t)$ is Laplace transformable) and $\mathcal{L}^{-1}\{F(s)\}$ denotes the inverse Laplace transform of $F(s)$ (provided it exists).

\section{Model Reduction fOR Linear time-Delay SYSTEMS}

In this section we derive a model reduction theory for linear differential time-delay systems. To keep the notation as simple as possible we begin with the class of systems with discretedelays and we then discuss the extension of the results to more general types of delays and representations in Section II-D.

\section{A. Definition of $\Pi$ : linear time-delay systems}

Consider a linear, single-input, single-output, continuoustime, time-delay system with constant discrete-delays described by the equations ${ }^{1}$

$$
\begin{array}{ll}
\dot{x}=\sum_{j=0}^{\varsigma} A_{j} x_{\tau_{j}}+\sum_{j=\varsigma+1}^{\mu} B_{j} u_{\tau_{j}}, & y=\sum_{j=0}^{\varsigma} C_{j} x_{\tau_{j}}, \\
x(\theta)=\phi(\theta), & -T \leq \theta \leq 0,
\end{array}
$$

with $x(t) \in \mathbb{R}^{n}, u(t) \in \mathbb{R}, y(t) \in \mathbb{R}, \phi \in \mathfrak{R}_{T}^{n}, A_{j} \in \mathbb{R}^{n \times n}$ and $C_{j} \in \mathbb{R}^{1 \times n}$ with $^{2} j=0, \ldots, \varsigma, B_{j} \in \mathbb{R}^{n \times 1}$ with $j=$ $\varsigma+1, \ldots, \mu, \tau_{0}=0$ and $\tau_{j} \in \mathbb{R}_{>0}$ with $j=1, \ldots, \mu$. Let

$$
W(s)=\bar{C}(s)(s I-\bar{A}(s))^{-1} \bar{B}(s),
$$

with

$$
\begin{aligned}
\bar{A}(s) & =\sum_{j=0}^{\varsigma} A_{j} e^{-s \tau_{j}}, \quad \bar{B}(s)=\sum_{j=\varsigma+1}^{\mu} B_{j} e^{-s \tau_{j}}, \\
\bar{C}(s) & =\sum_{j=0}^{\varsigma} C_{j} e^{-s \tau_{j}},
\end{aligned}
$$

be the associated transfer function and assume (1) is minimal, i.e., controllable and observable. We begin defining the moments of system (1) at some $s_{i} \in \mathbb{C}$ and showing that there exists a one-to-one relation between the moments and the (unique) solution of a Sylvester-like equation. We introduce the following assumptions frequently referred to in the paper.

Definition 1. Let $s_{i} \in \mathbb{C} \backslash \sigma(\bar{A}(s))$. The 0-moment of system (1) at $s_{i}$ is the complex number

$$
\eta_{0}\left(s_{i}\right)=\bar{C}\left(s_{i}\right)\left(s_{i} I-\bar{A}\left(s_{i}\right)\right)^{-1} \bar{B}\left(s_{i}\right) .
$$

The k-moment of system (1) at $s_{i}$ is the complex number

$$
\eta_{k}\left(s_{i}\right)=\frac{(-1)^{k}}{k !}\left[\frac{d^{k}}{d s^{k}} W(s)\right]_{s=s_{i}},
$$

with $k \geq 1$ integer.

This definition of moment is justified by the fact that the $k$-moment of a linear system at $s_{i}$ is defined as the $k$-th coefficient of the Laurent series expansion of the transfer function $W(s)$ at $s_{i} \in \mathbb{C}$ (see [1, Chapter 11]), provided it exists.

\footnotetext{
${ }^{1}$ The results can be extended to multi-input, multi-output (MIMO) systems straightforwardly. The problem in the MIMO case is called tangential interpolation and is discussed, in the present context, in [20], see also [64].

${ }^{2}$ The delays of $A_{j}$ and $C_{j}$ are taken, without loss of generality, equal to ease the notation.
} 
Lemma 1. Consider system (1) and let $s_{i} \in \mathbb{C} \backslash \sigma(\bar{A}(s))$. Assume that $\widetilde{\Pi}$ is the unique solution of the Sylvester-like equation

$$
\sum_{j=0}^{\varsigma} A_{j} \widetilde{\Pi} e^{-\Sigma_{k} \tau_{j}}+\sum_{j=\varsigma+1}^{\mu} B_{j} L_{k} e^{-\Sigma_{k} \tau_{j}}=\widetilde{\Pi} \Sigma_{k},
$$

with $L_{k}=\left[\begin{array}{llll}1 & 0 & \ldots & 0\end{array}\right] \in \mathbb{R}^{(k+1)}$ and

$$
\Sigma_{k}=\left[\begin{array}{ccccc}
s_{i} & 1 & 0 & \ldots & 0 \\
0 & s_{i} & 1 & \ldots & 0 \\
\vdots & \vdots & \ddots & \ddots & \vdots \\
0 & \ldots & 0 & s_{i} & 1 \\
0 & \ldots & \ldots & 0 & s_{i}
\end{array}\right] \in \mathbb{R}^{(k+1) \times(k+1)}
$$

Then

$$
\left[\begin{array}{lll}
\eta_{0}\left(s_{i}\right) & \ldots & \eta_{k}\left(s_{i}\right)
\end{array}\right]=\sum_{j=0}^{\varsigma} C_{j} \widetilde{\Pi} e^{-\Sigma_{k} \tau_{j}} \Psi_{k},
$$

where $\Psi_{k}=\operatorname{diag}\left(1,-1,1, \ldots,(-1)^{k}\right) \in \mathbb{R}^{(k+1) \times(k+1)}$.

Proof. Let $\widetilde{\Pi}=\left[\begin{array}{llll}\widetilde{\Pi}_{0} & \widetilde{\Pi}_{1} & \ldots & \widetilde{\Pi}_{k}\end{array}\right]$. Since $\Sigma_{k}$ is in Jordan form then

$$
e^{-\Sigma_{k} \tau_{j}}=e^{-s_{i} \tau_{j}}\left[\begin{array}{ccccc}
1 & \tau_{j} & \frac{\tau_{j}^{2}}{2} & \cdots & \frac{\tau_{j}^{k-1}}{(k-1) !} \\
0 & 1 & \tau_{j} & \cdots & \frac{\tau_{j}^{k-2}}{(k-2) !} \\
\vdots & \vdots & \ddots & \ddots & \vdots \\
0 & \ldots & 0 & 1 & \tau_{j} \\
0 & \ldots & \ldots & 0 & 1
\end{array}\right] .
$$

Thus, the first column of equation (4) can be rewritten as

$$
\sum_{j=0}^{\varsigma} A_{j} \widetilde{\Pi}_{0} e^{-s_{i} \tau_{j}}+\sum_{j=\varsigma+1}^{\mu} B_{j} e^{-s_{i} \tau_{j}}=\widetilde{\Pi}_{0} s_{i},
$$

the second column can be rewritten as

$$
\begin{aligned}
\sum_{j=0}^{\varsigma} A_{j} e^{-s_{i} \tau_{j}} \widetilde{\Pi}_{1} & +\sum_{j=0}^{\varsigma}-\tau_{j} A_{j} e^{-s_{i} \tau_{j}} \widetilde{\Pi}_{0}- \\
& -\sum_{j=\varsigma+1}^{\mu} \tau_{j} B_{j} e^{-s_{i} \tau_{j}}=\widetilde{\Pi}_{1} s_{i}+\widetilde{\Pi}_{0},
\end{aligned}
$$

and so on until the last column

$$
\begin{array}{r}
\sum_{l=k}^{0} \sum_{j=0}^{\varsigma} A_{j} \widetilde{\Pi}_{k-l} \frac{\left(-\tau_{j}\right)^{l}}{l !} e^{-s_{i} \tau_{j}}+\sum_{j=\varsigma+1}^{\mu} B_{j} \frac{\left(-\tau_{j}\right)^{k}}{k !} e^{-s_{i} \tau_{j}}= \\
=\widetilde{\Pi}_{k} s_{i}+\widetilde{\Pi}_{k-1} .
\end{array}
$$

As a result, $\widetilde{\Pi}_{0}$ can be determined from equation (5) as

$$
\begin{aligned}
\widetilde{\Pi}_{0} & =\left(s_{i} I-\sum_{j=0}^{\varsigma} A_{j} e^{-s_{i} \tau_{j}}\right)^{-1} \sum_{j=\varsigma+1}^{\mu} B_{j} e^{-s_{i} \tau_{j}}= \\
& =\left(s_{i} I-\bar{A}\left(s_{i}\right)\right)^{-1} \bar{B}\left(s_{i}\right),
\end{aligned}
$$

$\widetilde{\Pi}_{1}$ from equation (6) and $\widetilde{\Pi}_{0}$ as

$$
\widetilde{\Pi}_{1}=-\left(s_{i} I-\sum_{j=0}^{\varsigma} A_{j} e^{-s_{i} \tau_{j}}\right)^{-1}\left(I+\sum_{j=1}^{\varsigma} \tau_{j} A_{j} e^{-s_{i} \tau_{j}}\right) \times
$$

$$
\begin{aligned}
& \times\left(s_{i} I-\sum_{j=0}^{\varsigma} A_{j} e^{-s_{i} \tau_{j}}\right)^{-1} \sum_{j=\varsigma+1}^{\mu} B_{j} e^{-s_{i} \tau_{j}}+ \\
& -\left(s_{i} I-\sum_{j=0}^{\varsigma} A_{j} e^{-s_{i} \tau_{j}}\right)^{-1} \sum_{j=\varsigma+1}^{\mu} \tau_{j} B_{j} e^{-s_{i} \tau_{j}}= \\
& =\left[\frac{d}{d s}\left((s I-\bar{A}(s))^{-1} \bar{B}\left(s_{i}\right)\right)\right]_{s=s_{i}} .
\end{aligned}
$$

Iterating for all $k$, yields

$$
\widetilde{\Pi}_{k}=\frac{1}{k !}\left[\frac{d^{k}}{d s^{k}}\left((s I-\bar{A}(s))^{-1} \bar{B}\left(s_{i}\right)\right)\right]_{s=s_{i}} .
$$

Finally, exploiting the columns of $\widetilde{\Pi}$, the moments can be written as

$$
\begin{aligned}
& \sum_{j=0}^{\varsigma} C_{j} \widetilde{\Pi} e^{-\Sigma_{k} \tau_{j}}= \\
& =\left[\begin{array}{lll}
\sum_{j=0}^{\varsigma} C_{j} \widetilde{\Pi}_{0} e^{-s_{i} \tau_{j}} & \ldots & \sum_{l=k}^{0} \sum_{j=0}^{\varsigma} C_{j} \widetilde{\Pi}_{k-l} \frac{\left(-\tau_{j}\right)^{l}}{l !} e^{-s_{i} \tau_{j}}
\end{array}\right] \\
& =\left[\begin{array}{lll}
\bar{C}\left(s_{i}\right) \widetilde{\Pi}_{0} & \ldots & \sum_{l=k}^{0} \frac{1}{l !} \frac{d^{l}}{d s^{l}}[\bar{C}(s)]_{s=s_{i}} \widetilde{\Pi}_{k-l}
\end{array}\right] \\
& =\left[\begin{array}{lll}
\eta_{0}\left(s_{i}\right) & \ldots & (-1)^{k} \eta_{k}\left(s_{i}\right)
\end{array}\right],
\end{aligned}
$$

which proves the claim.

Equation (4) can be written eliminating the fact that $\Sigma_{k}$ and $L_{k}$ have a special structure. As a result the following holds.

Theorem 1. Consider system (1) and let $s_{i}$, with $i=1, \ldots, \eta$, be a set of numbers such that $s_{i} \in \mathbb{C} \backslash \sigma(\bar{A}(s))$. Let $S \in \mathbb{R}^{\nu \times \nu}$ be any non-derogatory matrix with characteristic polynomial

$$
p(s)=\prod_{i=1}^{\eta}\left(s-s_{i}\right)^{k_{i}},
$$

where $\nu=\sum_{i=1}^{\eta} k_{i}$, and $L$ be such that the pair $(L, S)$ is observable. Assume that $\Pi$ is the unique solution of the Sylvester-like equation

$$
\sum_{j=0}^{\varsigma} A_{j} \Pi e^{-S \tau_{j}}-\Pi S=-\sum_{j=\varsigma+1}^{\mu} B_{j} L e^{-S \tau_{j}} .
$$

Then there exists a one-to-one relation between the moments $\eta_{0}\left(s_{1}\right), \ldots, \eta_{k_{1}-1}\left(s_{1}\right), \ldots, \eta_{0}\left(s_{\eta}\right), \ldots, \eta_{k_{\eta}-1}\left(s_{\eta}\right)$ and the matrix $\sum_{j=0}^{\varsigma} C_{j} \Pi e^{-S \tau_{j}}$.

Proof. Note that it is sufficient to prove the claim for $\eta=1$. By observability of the pair $(L, S)$ there is a unique invertible matrix $T$ such that $S=T^{-1} \Sigma_{k} T$ and $L=L_{k} T$. Then equation (9) becomes

$$
\begin{aligned}
\sum_{j=0}^{\mu} A_{j} \Pi e^{-\left(T^{-1} \Sigma_{k} T\right) \tau_{j}}-\Pi T^{-1} \Sigma_{k} T & = \\
& -\sum_{j=\varsigma+1}^{\mu} B_{j} L e^{-\left(T^{-1} \Sigma_{k} T\right) \tau_{j}} .
\end{aligned}
$$


The claim follows defining $\widetilde{\Pi}=\Pi T^{-1}$, recalling that $e^{T^{-1} X T}=T^{-1} e^{X} T$ and that the moments are coordinates invariant.

\section{B. Solution of the Sylvester-like equation}

Equation (9) is a Sylvester equation only if $\varsigma=0$. Nevertheless, it is a linear equation in $\Pi$ and it can be solved with the use of the vectorization operator and the Kronecker product. To this end, it is necessary to determine when the equation admits a unique solution. In this section we solve this problem in the general case and for two special cases.

Lemma 2. Equation (9) has a unique solution if and only if $s_{i} \in \mathbb{C} \backslash \sigma(\bar{A}(s))$ for all $i=1, \ldots, \eta$.

Proof. Suppose, without loss of generality, that the matrix $S$ is in complex Jordan form. Then the matrices $S^{\top}$ and $e^{-S^{\top} \tau_{j}}$ are lower triangular and their $i$-th eigenvalue is $s_{i}$ and $e^{-s_{i} \tau_{j}}$, respectively. We recall that the eigenvalues of the sum of lower triangular matrices is the sum of the eigenvalues. The claim follows since equation (9) has a unique solution (see [58], [59]) if and only if

$$
\operatorname{det}\left(\sum_{j=0}^{\varsigma}\left(e^{-S^{\top} \tau_{j}} \otimes A_{j}\right)-S^{\top} \otimes I\right) \neq 0,
$$

which holds if and only if

$$
\prod_{i=1}^{\eta} \operatorname{det}\left(\sum_{j=0}^{\varsigma} A_{j} e^{-s_{i} \tau}-s_{i} I\right) \neq 0
$$

hence the claim.

Corollary 1. Equation (9) has a unique solution if the following holds.

- $A_{0}=0, A_{1} \neq 0, \mu=\varsigma=1$, and $\sigma\left(A_{1}\right) \cap \sigma\left(S e^{S \tau}\right)=\emptyset$.

- The matrices $A_{j}$ for $j=0,1, \ldots, \varsigma$ commute and $\sum_{j=0}^{\mu} e^{-s_{l} \tau_{j}} \lambda_{j i} \neq s_{l}$ for $i=1, \ldots, n$ and $l=1, \ldots, \eta$, with $\lambda_{j i}$ and $s_{l}$ eigenvalues of $A_{j}$ and $S$, respectively.

Proof: The claim is a direct consequence of the use of the vectorization operator (see [58], [59]).

\section{Definition of $\Pi$ : linear time-delay systems - Revisited}

To prepare the ground for the study of nonlinear timedelay systems, in this section we revisit the "interpolationbased" description of moment developed just now and give the equivalent "steady-state-based" description using the center manifold theory. For an extensive analysis of the relation between the two descriptions see [21] and also [17]. The center manifold theory for time-delay systems has been widely studied. The results in [61] establish that the theory for finite dimensional systems can be extended to infinite dimensional systems (and then to time-delay systems). In particular, as for finite dimensional systems, if the linearized system has $q$ eigenvalues on the imaginary axis then there exists a $q$ dimensional local integral manifold (referred to as center manifold) for the original system. In addition, the well-defined restriction of the dynamics of the system to the manifold is finite dimensional. An overview on the center manifold theory for time-delay systems has been given in [22] and references therein.

Theorem 2. Let $S \in \mathbb{R}^{\nu \times \nu}$ be any non-derogatory matrix with characteristic polynomial (8). Consider system (1) and assume $s_{i} \in \mathbb{C} \backslash \sigma(\bar{A}(s))$, with $i=1, \ldots, \eta$, and $\sigma(\bar{A}(s)) \subset \mathbb{C}_{<0}$. Consider the interconnection of system (1) with the system

$$
\dot{\omega}=S \omega, \quad u=L \omega
$$

with $L$ and $\omega(0)$ such that the triple $(L, S, \omega(0))$ is minimal. Then there exists a one-to-one ${ }^{3}$ relation between the moments $\eta_{0}\left(s_{1}\right), \ldots, \eta_{k_{1}-1}\left(s_{1}\right), \ldots, \eta_{0}\left(s_{\eta}\right), \ldots, \eta_{k_{\eta}-1}\left(s_{\eta}\right)$ and the steady-state response of the output of such interconnected system.

Proof. Consider the interconnection of system (1) with system (10). By the assumptions on $\sigma(\bar{A}(s))$ and $\sigma(S)$, the interconnected system has a globally well-defined invariant manifold given by $\mathcal{M}=\left\{(x, \omega) \in \mathbb{R}^{n+\nu}: x=\Pi \omega\right\}$, with $\Pi$ the unique solution of the Sylvester-like equation (9). We prove now that $\mathcal{M}$ is attractive. Consider the equation

$$
\begin{aligned}
& \overbrace{x-\Pi \omega}^{-\varsigma}= \\
& =\sum_{j=0}^{\varsigma} A_{j} x_{\tau_{j}}+\sum_{j=\varsigma+1}^{\mu} B_{j} L \omega_{\tau_{j}}-\Pi S \omega= \\
& =\sum_{j=0}^{\varsigma} A_{j} x_{\tau_{j}}+\left(\sum_{j=\varsigma+1}^{\mu} B_{j} L e^{-S \tau_{j}}-\Pi S\right) \omega=
\end{aligned}
$$

in which we used the fact that $\omega\left(t-\tau_{j}\right)=e^{-S \tau_{j}} \omega(t)$, substituting (9) in the last line, yields

$$
\overbrace{x-\Pi \omega}^{-\varsigma}=\sum_{j=0}^{\varsigma} A_{j}\left(x_{\tau_{j}}-\Pi \omega_{\tau_{j}}\right)
$$

Computing the Laplace transform on both sides yields

$$
\begin{aligned}
& s(X(s)-\Pi \Omega(s))-(x(0)-\Pi \omega(0))= \\
& =\left(\sum_{j=0}^{\varsigma} A_{j} e^{-s \tau_{j}}\right)(X(s)-\Pi \Omega(s)),
\end{aligned}
$$

and, by the assumptions on $\sigma(\bar{A}(s))$, we have

$$
X(s)-\Pi \Omega(s)=(s I-\bar{A}(s))^{-1}(x(0)-\Pi \omega(0)) .
$$

Finally, computing the inverse Laplace transform, yields

$$
x(t)-\Pi \omega(t)=\mathcal{L}^{-1}\left\{(s I-\bar{A}(s))^{-1}(x(0)-\Pi \omega(0))\right\} .
$$

\footnotetext{
${ }^{3}$ By one-to-one relation we mean that the moments are uniquely determined by the steady-state response of $y(t)$ and vice versa.
} 
Since $\sigma(\bar{A}(s)) \subset \mathbb{C}_{<0}$, by [22, Chapter 1, Theorem 6.2], $\mathcal{M}$ is attractive. As a result

$$
\begin{aligned}
y(t) & =\sum_{j=0}^{\varsigma} C_{j}\left(x_{\tau_{j}}-\Pi \omega_{\tau_{j}}\right)+\sum_{j=0}^{\varsigma} C_{j} \Pi \omega_{\tau_{j}} \\
& =\sum_{j=0}^{\varsigma} C_{j} \Pi \omega_{\tau_{j}}+\sum_{j=0}^{\varsigma} C_{j} \mathcal{L}^{-1}\left\{(s I-\bar{A}(s))^{-1}(x(0)-\Pi \omega(0))\right\} \\
& =\sum_{j=0}^{\varsigma} C_{j} \Pi e^{-S \tau_{j}} \omega+\varepsilon(t),
\end{aligned}
$$

where $\sum_{j=0}^{\varsigma} C_{j} \Pi e^{-S \tau_{j}} \omega(t)$ describes the steady-state response, whereas

$$
\varepsilon(t)=\sum_{j=0}^{\varsigma} C_{j} \mathcal{L}^{-1}\left\{(s I-\bar{A}(s))^{-1}(x(0)-\Pi \omega(0))\right\},
$$

describes the transient response which vanishes exponentially. This proves the claim.

Exploiting Theorem 2, in [65] the problem of model reduction for linear delay-free and linear time-delay systems from input/output data is addressed. Therein, an algorithm that, given the signal $\omega$ and the output $y$, retrieves the moments of a system for which the matrices $A, B, C$ and $D$ are not known is devised. However, note that the importance of Theorem 2 goes beyond the simple computation of the moments because it highlights the relation between the steady-state response and the moments.

\section{A general class of linear time-delay systems}

All the results presented for discrete-delays can be generalized to linear differential time-delay systems with distributeddelays and linear neutral differential time-delay systems. The moments of any type of linear differential time-invariant time-delay system can be characterized with a Sylvester-like equation given an assumption similar to $s_{i} \in \mathbb{C} \backslash \sigma(\bar{A}(s))$. Consider a linear, single-input, single-output, continuous-time, neutral time-delay system with discrete-delays and distributeddelays described by the equations

$$
\begin{gathered}
\dot{x}=\sum_{j=1}^{q} D_{j} \dot{x}_{c_{j}}+\sum_{j=0}^{\varsigma} A_{j} x_{\tau_{j}}+\sum_{j=\varsigma+1}^{\mu} B_{j} u_{\tau_{j}}+ \\
+\sum_{j=1}^{r} \int_{t-h_{j}}^{t}\left(G_{j} x(\theta)+H_{j} u(\theta)\right) d \theta, \\
\begin{array}{ll}
y=\sum_{j=0}^{\varsigma} C_{j} x_{\tau_{j}}, & -T \leq \theta \leq 0, \\
x(\theta)=\phi(\theta), & -T \leq \theta
\end{array}
\end{gathered}
$$

with $x(t) \in \mathbb{R}^{n}, u(t) \in \mathbb{R}, y(t) \in \mathbb{R}, \phi \in \mathfrak{R}_{T}^{n}, A_{j} \in \mathbb{R}^{n \times n}$ and $C_{j} \in \mathbb{R}^{1 \times n}$ with $j=0, \ldots, \varsigma, B_{j} \in \mathbb{R}^{n \times 1}$ with $j=$ $\varsigma+1, \ldots, \mu, D_{j} \in \mathbb{R}^{n \times n}$ with $j=1, \ldots, q, G_{j} \in \mathbb{R}^{n \times n}$ and $H_{j} \in \mathbb{R}^{n \times 1}$ with $j=1, \ldots, r, \tau_{0}=0, \tau_{j} \in \mathbb{R}_{>0}$ with $j=1, \ldots, \mu, c_{j} \in \mathbb{R}_{>0}$ with $j=1, \ldots, q$ and $h_{j} \in \mathbb{R}_{>0}$ with $j=1, \ldots, r$. The transfer function $W(s)$ is defined by (2) with

$$
\begin{aligned}
\bar{A}(s) & =\sum_{j=1}^{q} D_{j} s e^{-s c_{j}}+\sum_{j=0}^{\varsigma} A_{j} e^{-s \tau_{j}}+\sum_{j=1}^{r} G_{j} \frac{1-e^{-s h_{j}}}{s}, \\
\bar{B}(s) & =\sum_{j=\varsigma+1}^{\mu} B_{j} e^{-s \tau_{j}}+\sum_{j=1}^{r} H_{j} \frac{1-e^{-s h_{j}}}{s}, \\
\bar{C}(s) & =\sum_{j=0}^{\varsigma} C_{j} e^{-s \tau_{j}} .
\end{aligned}
$$

We introduce the following stability condition (i.e. a "formal stability" condition, see [66], [31]).

Assumption 1. Assume the difference equation

$$
x(t)+\sum_{j=1}^{q} D_{j} x\left(t-\omega_{j}\right)=0
$$

is asymptotically stable.

Theorem 3. Assume $0 \notin \sigma(S)$. Theorem 1 holds, with the same assumptions, for system (11) replacing equation (9) with

$$
\begin{aligned}
& \sum_{j=0}^{\varsigma} A_{j} \Pi e^{-S \tau_{j}}+\sum_{j=1}^{r} G_{j} \Pi S^{-1}\left(I-e^{-S h_{j}}\right)+ \\
& +\sum_{j=1}^{q} D_{j} \Pi S e^{-S c_{j}}-\Pi S= \\
& \quad=-\sum_{j=\varsigma+1}^{\mu} B_{j} L e^{-S \tau_{j}}-\sum_{j=1}^{r} H_{j} L S^{-1}\left(I-e^{-S h_{j}}\right) .
\end{aligned}
$$

Proof. The proof is similar to that of Theorem 1, hence it is omitted.

Theorem 4. Assume $0 \notin \sigma(S)$ and Assumption 1 holds. Theorem 2 holds, with the same assumptions, for system (11).

Proof. The claim can be proved noting that $\sigma(\bar{A}(s)) \subset \mathbb{C}_{<0}$, with the definitions in (12), and Assumption 1 guarantee asymptotic stability of system (11) [67], [31]. The additional assumption that $S$ is invertible is necessary because in equation (13) the distributed-delays generate terms in $S^{-1}$.

Remark 1. As noted in [68], many systems can be described by the equations (11) with, in most cases, a single neutral delay, i.e. $q=1$. In this case Assumption 1 holds if $\sigma\left(D_{1}\right) \subset$ $\mathbb{D}_{<1}$, see [22] and [69].

Remark 2. Since hyperbolic partial differential equations can be locally expressed as neutral time-delay systems and, conversely, any time-delay $y(t)=u(t-\tau)$ can be represented by a classical transport equation (see [70], [24], [31]), the techniques presented in the paper can be used to establish a model reduction theory for some classes of partial differential equations: the example studied in [63] is, in fact, a neutral system originated from a partial differential equation. A similar remark applies for other relations, such as the ones established between time-delay systems and fractional derivation equations [71]. 
To keep the notation light only the discrete-delay case is considered in the remaining of the paper. However, the extension of the following results to system (11) is straightforward.

\section{E. Reduced order model for linear time-delay systems}

In this and the following sections a family of systems achieving moment matching is presented and the possibility of interpolating a larger number of points maintaining the same "number of equations" is investigated.

Definition 2. Consider system (1) and let $S \in \mathbb{R}^{\nu \times \nu}$ be any non-derogatory matrix with characteristic polynomial (8). Let $s_{i} \in \mathbb{C} \backslash \sigma(\bar{A}(s))$ for all $i=1, \ldots, \eta$, and let $L$ be such that the pair $(L, S)$ is observable. Then the system

$$
\dot{\xi}=\sum_{j=0}^{\varrho} F_{j} \xi_{\chi_{j}}+\sum_{j=\varrho+1}^{\rho} G_{j} u_{\chi_{j}}, \quad \psi=\sum_{j=0}^{d} H_{j} \xi_{\chi_{j}}
$$

with $\xi(t) \in \mathbb{R}^{\nu}, \psi(t) \in \mathbb{R}, F_{j} \in \mathbb{R}^{\nu \times \nu}$ for $j=0, \ldots, \varrho$, $G_{j} \in \mathbb{R}^{\nu \times 1}$ for $j=\varrho+1, \ldots, \rho, H_{j} \in \mathbb{R}^{1 \times \nu}$ for $j=0, \ldots, k$, $\chi_{0}=0$ and $\chi_{j} \in \mathbb{R}_{>0}$ for $j=1, \ldots, \max \{\rho, d\}$, is a model of system (1) at $S$, if there exists a unique solution $P$ of the equation

$$
\sum_{j=0}^{\varrho} F_{j} P e^{-S \chi_{j}}-P S=-\sum_{j=\varrho+1}^{\rho} G_{j} L e^{-S \chi_{j}}
$$

such that

$$
\sum_{j=0}^{\varsigma} C_{j} \Pi e^{-S \tau_{j}}=\sum_{j=0}^{d} H_{j} P e^{-S \chi_{j}},
$$

where $\Pi$ is the unique solution of (9). System (14) is a reduced order model of system (1) at $S$ if $\nu<n$, or if $\varrho<\varsigma$, or if $\rho<\mu$, or if $d<\varsigma$.

Note that equation (15) defines the moments of the reduced order model, whereas equation (16) gives the matching condition between the moments of the system to be reduced and the moments of the reduced order model. To assure that the solution $P$ of equation (15) exists and is unique and that equation (16) have a solution, we need an additional assumption on the reduced order model.

Lemma 3. Let $\bar{F}(s)=\sum_{j=0}^{\varrho} F_{j} e^{-s \chi_{j}}$. Equation (15) has a unique solution $P$ and equation (16) has a solution uniquely determined by the matrices $H_{j}$ if and only if $s_{i} \notin \sigma(\bar{F}(s))$ for all $i=1, \ldots, \eta$ and the pair $(L, S)$ is observable.

Proof. The uniqueness of the solution $P$ of equation (15) follows from the arguments given in Section II-B. The observability of the pair $(L, S)$ guarantees that $P$ is full rank and, as a consequence, (16) can always be solved.

\section{F. Reduced order model with free $F_{j}$}

To construct a family of models that achieves moment matching at $\nu$ points one could select

$$
\begin{aligned}
& F_{0}=S-\sum_{j=\varrho+1}^{\rho} G_{j} L e^{-S \chi_{j}}-\sum_{j=1}^{\varrho} F_{j} e^{-S \chi_{j}} \\
& H_{0}=\sum_{j=0}^{\varsigma} C_{j} \Pi e^{-S \tau_{j}}-\sum_{j=1}^{d} H_{j} e^{-S \chi_{j}},
\end{aligned}
$$

and note that this selection solves equations (15), (16) for $P=$ $I$. This yields the family of reduced order models described by the equations

$$
\begin{aligned}
\dot{\xi}= & \left(S-\sum_{j=\varrho+1}^{\rho} G_{j} L e^{-S \chi_{j}}-\sum_{j=1}^{\varrho} F_{j} e^{-S \chi_{j}}\right) \xi+ \\
& +\sum_{j=1}^{\varrho} F_{j} \xi_{\chi_{j}}+\sum_{j=\varrho+1}^{\rho} G_{j} u_{\chi_{j}}, \\
\psi= & \left(\sum_{j=0}^{\varsigma} C_{j} \Pi e^{-S \tau_{j}}-\sum_{j=1}^{d} H_{j} e^{-S \chi_{j}}\right) \xi+\sum_{j=1}^{d} H_{j} \xi_{\chi_{j}},
\end{aligned}
$$

with $G_{j}$ and $F_{j}$ any matrices such that $s_{i} \notin \sigma(\bar{F}(s))$, for all $i=1, \ldots, \eta$.

The proposed model has several free design parameters, namely $G_{j}, F_{j}, H_{j}, \chi_{j}, \varrho, \rho$ and $d$. We note that selecting $\varrho=0, \rho=1, d=0$ and $\chi_{1}=0$ (in this case we define $G=G_{1}$ ), yields a reduced order model with no delays. In other words, we reduce an infinite dimensional system to a finite dimensional one, of dimension $\nu$. This reduced order model coincides with the one in [17] and all results therein are directly applicable: the parameter $G$ can be selected to achieve matching with prescribed eigenvalues, matching with prescribed relative degree, etc.

Remark 3. The problem of reducing an infinite dimensional system to a finite dimensional one is not new in literature, see e.g. [72], [56] and [57], and is how the model reduction has been traditionally intended for time-delay systems, see $e . g$. [42], [43], [44], [45], [46], [47], [48], [49], [50], [51], [52] and [73], in which the problem of reducing the transfer function of a linear time-delay system to a rational function is studied. A variety of methods are used, e.g. Padé approximation, Taylor expansions, spline approximations and Hankel operator.

On the other hand, the choice of eliminating the delays is likely to destroy some underlying dynamics of the model and, as shown in [38], [39], [40], [41] and [60], delays are not always detrimental (for example to stability). With this in mind, a possible choice is to keep $F_{j}, G_{j}$ and $H_{j}$ free with $\varrho=d=\varsigma$ and $\rho=\mu$. In this case we can use the matrices $F_{j}, G_{j}$ and $H_{j}$, with $\tau_{j}=\chi_{j}$, to maintain some important physical properties of the delay structure of the system.

Example 1. To illustrate the above idea consider the example in Section 2.5 of [26] in which a model of a LC transmission line in considered. The linear neutral differential time-delay 
system is described by the equations

$$
\begin{aligned}
& \dot{x}=A x+D \dot{x}_{\tau}+B u, \\
& y=C x,
\end{aligned}
$$

with

$$
\begin{array}{rlrl}
A & =-\frac{1}{C_{1}}\left[\begin{array}{cc}
\frac{1}{R_{1}}+\sqrt{\frac{C_{0}}{L}} & 0 \\
-C_{1} & 0
\end{array}\right], D=\left[\begin{array}{rr}
0 & -\frac{2}{C_{1}} \sqrt{\frac{C_{0}}{L}} \alpha \\
0 & \alpha
\end{array}\right], \\
B & =\left[\begin{array}{ll}
b_{1} & b_{2}
\end{array}\right]^{\top}, & C & =\left[\begin{array}{ll}
c_{1} & c_{2}
\end{array}\right], \\
\alpha & =\frac{1-R_{0} \sqrt{\frac{C_{0}}{L}}}{1+R_{0} \sqrt{\frac{C_{0}}{L}}} & \tau & =2 \sqrt{L C_{0}},
\end{array}
$$

in which $C_{1} \in \mathbb{R}_{>0}, R_{1} \in \mathbb{R}_{>0}, C_{0} \in \mathbb{R}_{>0}, L \in \mathbb{R}_{>0}$, $R_{0} \in \mathbb{R}_{>0}, b_{1} \in \mathbb{R}, b_{2} \in \mathbb{R}, c_{1} \in \mathbb{R}$ and $c_{2} \in \mathbb{R}$. The system is such that if $R_{0} \sqrt{C_{0} / L}=1$ the delay part of the system disappears (a phenomenon called line-matching) and the model can be described by a system of ordinary differential equations. In the reduced model it is desirable to maintain this property to preserve the physical structure of the system. To simplify the example, suppose $S=1$ and $L=1$. Then the vector $\Pi$ can be computed from equation (13), that in this case is

$$
A \Pi+D \Pi e^{-\tau}-\Pi=-B,
$$

which has a unique solution if

$$
-\frac{1}{C_{1}}\left(\frac{1}{R_{1}}+\sqrt{\frac{C_{0}}{L}}\right) \neq 1, \quad e^{-\tau} \frac{1-R_{0} \sqrt{\frac{C_{0}}{L}}}{1+R_{0} \sqrt{\frac{C_{0}}{L}}} \neq 1 .
$$

Hence, a family of reduced order models, parameterized in $G$, is described by the equations

$$
\begin{aligned}
& \dot{\xi}=\left(1-e^{-\tau} \alpha-G\right) \xi+\alpha \dot{\xi}_{\tau}+G u, \\
& \psi=C \Pi \xi .
\end{aligned}
$$

Both equations (19) and (20) describe linear neutral differential time-delay systems when $R_{0} \sqrt{C_{0} / L} \neq 1$ and linear delay-free systems otherwise.

\section{G. Reduced order model interpolating at $(\varrho+1) \nu$ points}

The matrices $F_{j}$ and $H_{j}$ in (18) are design parameters. In this subsection we show how to exploit them to achieve moment matching at more than $\nu$ points, still maintaining the same dimension $\nu$ of the matrix $F_{0}$. We analyze the case in which $\varrho=1, \rho=3$ and $d=1\left(F_{1}, G_{2}, G_{3}\right.$ and $H_{1}$ are the free parameters), for ease of notation. We further assume without loss of generality that there are no delays in the equation of the output $y$ of system (1). The general case can be analyzed in a similar way.

Proposition 1. Let $S_{a} \in \mathbb{R}^{\nu \times \nu}$ and $S_{b} \in \mathbb{R}^{\nu \times \nu}$ be two nonderogatory matrices such that $\sigma\left(S_{a}\right) \cap \sigma\left(S_{b}\right)=\emptyset$ and let $L_{a}$ and $L_{b}$ be such that the pairs $\left(L_{a}, S_{a}\right)$ and $\left(L_{b}, S_{b}\right)$ are observable. Let $\Pi_{a}=\Pi$ be the unique solution of (9), with $L=L_{a}$ and $S=S_{a}$, and let $\Pi_{b}=\Pi$ be the unique solution of (9), with $L=L_{b}$ and $S=S_{b}$. Consider $F_{0}$ and $H_{0}$ as in (17) with $\chi_{2}=0, S=S_{a}$ and $L=L_{a}$.

- If $d=1$ and $L=L_{a}=L_{b}$, system (14) with the selection $F_{1}=\left(S_{b}-S_{a}-G_{3}\left(e^{-S_{b} \chi_{3}}-e^{-S_{a} \chi_{3}}\right)\right)\left(e^{-S_{b} \chi_{1}}-e^{-S_{a} \chi_{1}}\right)^{-1}$, $F_{0}=S_{a}-G_{2} L-G_{3} L e^{-S_{a} \chi_{3}}-F_{1} e^{-S_{a} \chi_{1}}$, $H_{1}=\left(C \Pi_{b}-C \Pi_{a}\right)\left(e^{-S_{b} \chi_{1}}-e^{-S_{a} \chi_{1}}\right)^{-1}$, $H_{0}=C \Pi_{a}-H_{1} e^{-S_{a} \chi_{1}}$,

belongs to the family (18) and is a reduced order model of system (1) achieving moment matching at $S_{a}$ and $S_{b}$, for any $G_{2}$ and $G_{3}$ such that $s_{i} \notin \sigma(\bar{F}(s))$, for all $i=$ $1, \ldots, \eta$.

- If $d=0$, the family (18) with

$$
\begin{aligned}
F_{1}=( & P_{b} S_{b}-S_{a} P_{b}+G_{2} L_{a} P_{b}+G_{3} L_{a} e^{-S_{b} \chi_{3}} P_{b} \\
& \left.-G_{2} L_{b}-G_{3} L_{b} e^{-S_{b} \chi_{3}}\right)\left(P_{b} e^{-S_{b} \chi_{1}}-e^{-S_{a} \chi_{1}} P_{b}\right)^{-1},
\end{aligned}
$$

is, for some $P_{b}$ such that $C \Pi_{a} P_{b}=C \Pi_{b}$, a reduced order model of system (1) achieving moment matching at $S_{a}$ and $S_{b}$, for any $G_{2}$ and $G_{3}$ such that $s_{i} \notin \sigma(\bar{F}(s))$, for all $i=1, \ldots, \eta$.

Proof. We begin with the case $d=1$. Easy computations show that

$$
\begin{aligned}
& F_{0}=S_{a}-G_{2} L_{a}-G_{3} L_{a} e^{-S_{a} \chi_{3}}-F_{1} e^{-S_{a} \chi_{1}}, \\
& H_{0}=C \Pi_{a}-H_{1} e^{-S_{a} \chi_{1}},
\end{aligned}
$$

defined in (17), solve

$$
\begin{aligned}
& F_{0} P_{a}+F_{1} P_{a} e^{-S_{a} \chi_{1}}-P_{a} S_{a}=-G_{2} L_{a}-G_{3} L_{a} e^{-S_{a} \chi_{3}}, \\
& C \Pi_{a}=H_{0} P_{a}+H_{1} P_{a} e^{-S_{a} \chi_{1}},
\end{aligned}
$$

with $P_{a}=I . F_{1}$ given in (22) solves the equation

$$
F_{0} P_{b}+F_{1} P_{b} e^{-S_{b} \chi_{1}}-P_{b} S_{b}=-G_{2} L_{b}-G_{3} L_{b} e^{-S_{b} \chi_{3}},
$$

for any invertible $P_{b}$. Substituting $H_{0}$ in

$$
C \Pi_{b}=H_{0} P_{b}+H_{1} P_{b} e^{-S_{b} \chi_{1}},
$$

yields

$$
H_{1}=\left(C \Pi_{b}-C \Pi_{a} P_{b}\right)\left(P_{b} e^{-S_{b} \chi_{1}}-e^{-S_{a} \chi_{1}} P_{b}\right)^{-1} .
$$

The matrices $F_{0}, F_{1}, H_{0}, H_{1}$ are such that the resulting reduced order model achieves moment matching at $S_{a}$ and $S_{b}$ and selecting $L=L_{a}=L_{b}$ and $P_{b}=I$ they yield (21). If $d=0$, equation (25) reduces to

$$
C \Pi_{b}=H_{0} P_{b}
$$

for some $P_{b}$. We then have to prove that there always exists a $P_{b}$ such that $C \Pi_{a} P_{b}=C \Pi_{b}$ and $F_{1}$ is well-defined. To prove the first claim note that the condition consists in finding $\nu^{2}$ parameters to solve $\nu$ equations. If $C \Pi_{a} \neq 0$ there exist always a $P_{b}$, full rank and upper triangular (possibly after a change of coordinates). Finally note that by the hypotheses on the system and the signal generator there exists at least a 


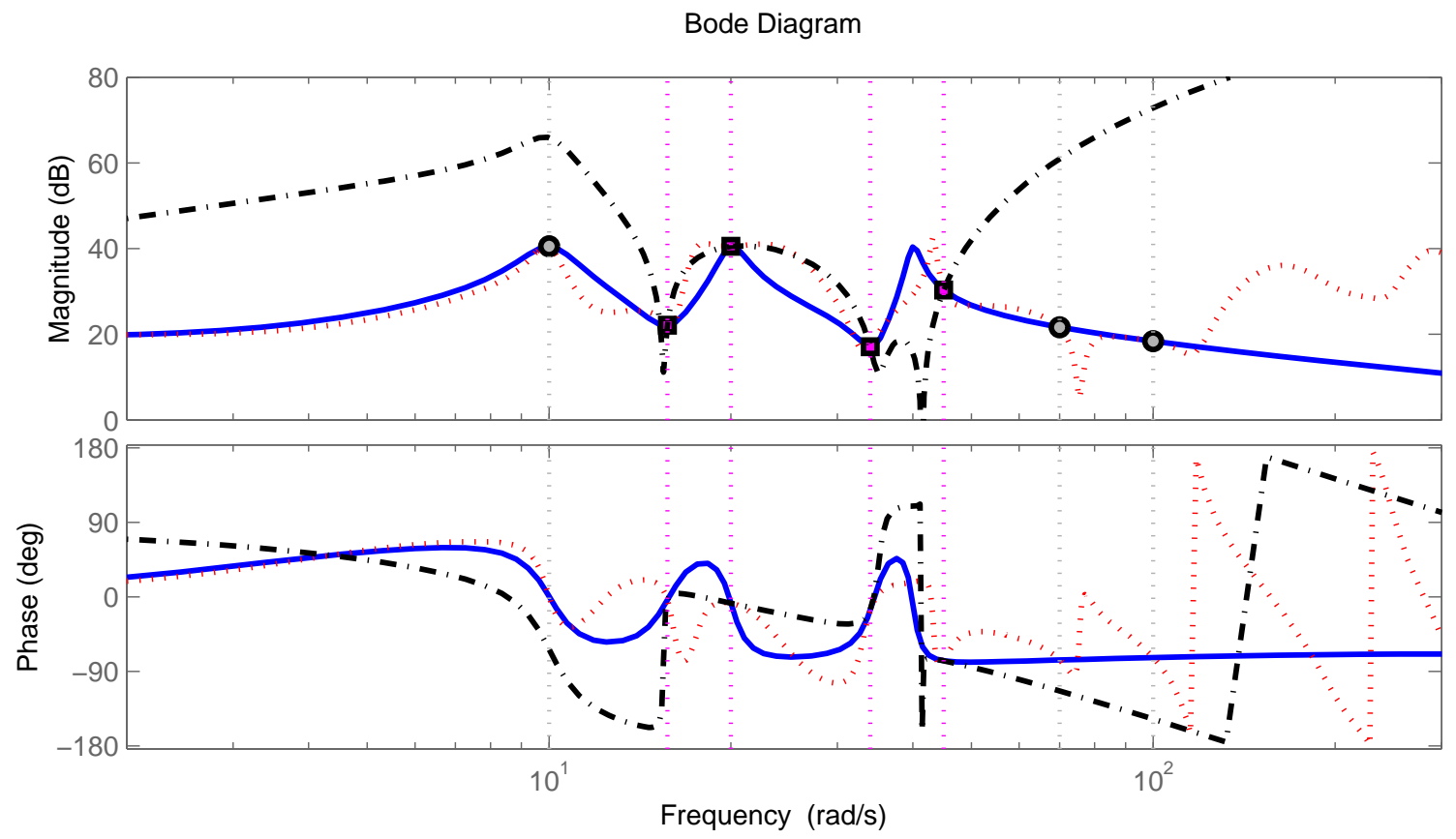

Fig. 1. Bode plot of the system (solid line), of the delay-free reduced order model (dash-dotted line) and of the time-delay reduced order model (dotted line). The squares indicate the first set of interpolation points, whereas the circles indicate the second set.

component of $C \Pi_{a}$ which is not zero. To prove the second claim we have to show that

$$
\operatorname{rank}\left\{P_{b} e^{-S_{b} \chi_{1}}-e^{-S_{a} \chi_{1}} P_{b}\right\}=\nu \text {. }
$$

Note now that selecting $S_{a}$ and $S_{b}$ in complex Jordan form implies that the matrices in equation (26) are all upper triangular. Condition (26) can be rewritten as

$$
\pi_{b i}\left(e^{-s_{b i} \chi_{1}}-e^{-s_{a i} \chi_{1}}\right) \neq 0, \quad \forall i=1, \ldots, \nu,
$$

with $\pi_{b i}, s_{a i}$ and $s_{b i}$ the eigenvalues of $P_{b}, S_{a}$ and $S_{b}$, respectively. Since $\sigma\left(S_{a}\right) \cap \sigma\left(S_{b}\right)=\emptyset$ then $\sigma\left(e^{-S_{a} \chi_{1}}\right) \cap$ $\sigma\left(e^{-S_{b} \chi_{1}}\right)=\emptyset$, hence the claim follows.

The family of systems characterized in Proposition 1 achieves moment matching at $2 \nu$ interpolation points. Note that the matrices $G_{2}$ and $G_{3}$ remain free parameters and they can be used to achieve the properties discussed in [17]. For instance $G_{2}$ and $G_{3}$ can be used to set both the eigenvalues of $F_{0}$ and $F_{1}$. In addition, note that $G_{j}$ has exactly $\nu$ free parameters. Hence, for instance, to assign the eigenvalues of $j F_{j}$ matrices, $j G_{j}$ matrices are needed. In [19], as already hinted in [17], it has been shown how to select $G$ (our $G_{2}$ ) to achieve the two-sided interpolation, i.e. how to exploit the free parameter $G$ to achieve interpolation at $2 \nu$ points. The two techniques may be combined and, in the case of Proposition 1, $G_{2}$ and $G_{3}$ may be selected to achieve interpolation at $4 \nu$ points.

Remark 4. The result can be generalized to $\varrho>1$ delays, obtaining a reduced order model that interpolates at $(\varrho+1) \nu$ points. This result can be used also when the system to be reduced is not a time-delay system. In other words, a system described by ordinary differential equations or time-delay differential equations can be reduced to a system described by time-delay differential equations with an arbitrary number of delays $\varrho$ achieving moment matching at $(\varrho+1) \nu$ points. This property of interpolating an arbitrary large number of points comes to the cost that the reduced order model becomes an infinite dimensional system. However, as noted in [38], [39], [40], [41] and [60], a reduced model with time delays may have better properties than one without delays.

Example 2. To illustrate the idea of approximating delayfree systems with time-delay systems exploiting the additional degrees of freedom to increase the number of interpolation points, we consider an example inspired by [74] (see also [1]). The example is a single-input, single-output system of order $n=1006$, which has a Bode plot with three peaks, described by the equations

$$
\dot{x}=A x+B u, \quad y=C x,
$$

where $A=\operatorname{diag}\left(A_{1}, A_{2}, A_{3}, A_{4}\right)$, with

$$
\begin{gathered}
A_{1}=\left[\begin{array}{cc}
-1 & 10 \\
-10 & -1
\end{array}\right], \quad A_{2}=\left[\begin{array}{cc}
-1 & 20 \\
-20 & -1
\end{array}\right], \\
A_{3}=\left[\begin{array}{cc}
-1 & 40 \\
-40 & -1
\end{array}\right], \quad A_{4}=\operatorname{diag}(-1,-2, \ldots,-1000),
\end{gathered}
$$

and

$$
B^{\top}=C=[\underbrace{10 \ldots 10}_{6 \text { times }} \underbrace{1 \ldots 1}_{1000 \text { times }}] .
$$

We start with obtaining a linear delay-free reduced order model of order $\nu=8$. The matrices of the signal generator (10) have been selected as $S=S_{a}=\operatorname{diag}\left(S_{2}, S_{3}, S_{4}, S_{5}\right)$, 
with $S_{2}=1.57 S_{1}, S_{3}=2 S_{1}, S_{4}=3.4 S_{1}, S_{5}=4.5 S_{1}$, where $S_{1}=A_{1}+I$, and $L$ randomly generated, to interpolate the moments close to the three peaks. The delayfree model (18) has been constructed with the technique presented in [17] assigning the eigenvalues of $F_{0}$ such that $\sigma\left(F_{0}\right) \subset \sigma(A)$. Fig. 1 shows the Bode plot of the system to be reduced (solid line) and of this reduced order model (dash-dotted line). The interpolation points are indicated by four squares. Note that the reduced order model approximates poorly the system because few interpolation points have been used (for comparison in [1] the order of the reduced order model is $\nu=11$, while in [65] $\nu=13$ ). We apply the technique presented in Proposition 1. The matrix $S_{b}$ has been selected as $S_{b}=\operatorname{diag}\left(S_{6}, S_{7}, S_{8}, S_{1}\right)$, with $S_{7}=7 S_{1}$, $S_{8}=10 S_{1}$ and

$$
S_{6}=\left[\begin{array}{ll}
0 & 1 \\
0 & 0
\end{array}\right] .
$$

Selecting $\chi=\chi_{1}=\chi_{3}=0.05$, yields

$$
F_{0}=S_{a}-\left(S_{b}-S_{a}\right)\left(e^{-S_{b} \chi}-e^{-S_{a} \chi}\right)^{-1} e^{-S_{a} \chi}-G_{2} L,
$$

and

$$
F_{1}=\left(S_{b}-S_{a}\right)\left(e^{-S_{b} \chi}-e^{-S_{a} \chi}\right)^{-1}-G_{3} L .
$$

Note that, because of the selection $\chi_{1}=\chi_{3}, F_{0}$ does not depend upon $G_{3}$. Thus, the eigenvalues of both $F_{0}$ and $F_{1}$ have been assigned such that $\sigma\left(F_{0}\right)=\sigma\left(F_{1}\right) \subset \sigma(A)$. In Fig. 1, the Bode plot of this reduced order model is represented by the dotted line. Three additional interpolation points are indicated with the circles. In addition the plot shows clearly that the model interpolates the point at zero, which is confirmed by a direct computation. Thus, the addition of one delay improved the quality of the approximation of the system without increasing the size of the matrices. However, note that a delay-free model with $\nu=16$ would be a better approximation because the introduction of the delay is, at the same time, detrimental (in particular at high frequencies).

Remark 5. Although it is possible to interpolate at several different points $s_{i}$ maintaining the same dimension $\nu$, the order of interpolation at $s_{i}$ cannot exceed $\nu$ because it is limited, by definition, by the dimension of the matrix $S_{j}$.

\section{MODEL REDUCTION FOR NONLINEAR TIME-DELAY SYSTEMS}

In this section we derive an extension of the model reduction method for nonlinear differential time-delay systems. To keep the notation simple we consider, without loss of generality, only delays (discrete or distributed) in the state and in the input, i.e. the output is delay-free. The neutral case is briefly discussed at the end of the section.

\section{A. Definition of П: nonlinear time-delay systems}

Consider a nonlinear, single-input, single-output, continuous-time, time-delay system described by the equations

$$
\begin{aligned}
& \dot{x}=f\left(x_{\tau_{0}}, \ldots, x_{\tau_{\varsigma}}, u_{\tau_{\varsigma}+1}, \ldots, u_{\tau_{\mu}}\right), \quad y=h(x), \\
& x(\theta)=\phi(\theta), \quad-T \leq \theta \leq 0,
\end{aligned}
$$

with $x(t) \in \mathbb{R}^{n}, u(t) \in \mathbb{R}, y(t) \in \mathbb{R}, \phi \in \mathfrak{R}_{T}^{n}, \tau_{0}=0$, $\tau_{j} \in \mathbb{R}_{>0}$ with $j=1, \ldots, \mu$ and $f$ and $h$ smooth mappings. Consider a signal generator described by the equations

$$
\dot{\omega}=s(\omega), \quad \theta=l(\omega),
$$

with $\omega(t) \in \mathbb{R}^{\nu}, \theta(t) \in \mathbb{R}, s$ and $l$ smooth mappings, and the interconnected system

$$
\begin{aligned}
& \dot{\omega}=s(\omega), \\
& \dot{x}=f\left(x_{\tau_{0}}, \ldots, x_{\tau_{\varsigma}}, l\left(\omega_{\tau_{\varsigma+1}}\right), \ldots, l\left(\omega_{\tau_{\mu}}\right)\right), \\
& y=h(x) .
\end{aligned}
$$

Suppose that $f(0, \ldots, 0,0, \ldots, 0)=0, s(0)=0, l(0)=0$ and $h(0)=0$.

Assumption 2. There exists a unique mapping $\pi(\omega)$, locally defined in a neighborhood of $\omega=0$, which solves the partial differential equation

$$
\frac{\partial \pi}{\partial \omega} s(\omega)=f\left(\pi\left(\bar{\omega}_{\tau_{0}}\right), \ldots, \pi\left(\bar{\omega}_{\tau_{\varsigma}}\right), l\left(\bar{\omega}_{\tau_{\varsigma}+1}\right), \ldots, l\left(\bar{\omega}_{\tau_{\mu}}\right)\right),
$$

where $\bar{\omega}_{\tau_{i}}=\Phi_{\tau_{i}}^{s}(\omega)$, with $i=0, \ldots, \mu$, is the flow of the vector field $s$ at $\tau_{i}$, see [75].

Assumption 2 implies that the interconnected system (29) possesses an invariant manifold, described by the equation $x=\pi(\omega)$. Note that the partial differential equation (30) is independent of time (as (9) and the correspondent equations given in [17]), e.g. if $s(\omega)=S \omega$ then $\bar{\omega}_{\tau_{i}}=e^{S \tau_{i}} \omega$.

Assumption 3. The signal generator (28) is observable, i.e. for any pair of initial conditions $\omega_{a}(0)$ and $\omega_{b}(0)$, such that $\omega_{a}(0) \neq \omega_{b}(0)$, the corresponding output trajectories $l\left(\omega_{a}(t)\right)$ and $l\left(\omega_{b}(t)\right)$ are such that $l\left(\omega_{a}(t)\right)-l\left(\omega_{b}(t)\right) \not \equiv 0$.

Definition 3. Consider system (27) and the signal generator (28). Suppose Assumption 2 and 3 hold. The function $h(\pi(\omega))$, with $\pi$ solution of equation (30), is the moment of system (27) at $(s(\omega), l(\omega))$.

Theorem 5. Consider system (27) and the signal generator (28). Suppose Assumption 3 holds. Assume the zero equilibrium of the system $\dot{x}=f\left(x_{\tau_{0}}, \ldots, x_{\tau_{\varsigma}}, 0, \ldots, 0\right)$ is locally exponentially stable and system (28) is Poisson stable ${ }^{4}$. Assume $\omega(0) \neq 0$. Then Assumption 2 holds and the moment of system $(27)$ at $(s(\omega), l(\omega))$ coincides with the steady-state response of the output of the interconnected system (29).

Proof. Under the stated assumptions there exist a well-defined center manifold described by $x=\pi(\omega)$. In addition, the assumptions on the signal generator guarantee that the steadystate response of the output is locally well-defined and it is described by $h(\pi(\omega))$ (see [76]).

\section{B. Reduced order model for nonlinear time-delay systems}

In this section a family of systems achieving moment matching is given.

\footnotetext{
${ }^{4}$ See [75, Chapter 8, page 388] for the definition of Poisson stability.
} 
Definition 4. Consider system (27) and the signal generator (28). Suppose Assumption 2 and 3 hold. Then the system

$$
\dot{\xi}=\phi\left(\xi_{\chi_{0}}, \ldots, \xi_{\chi_{\varrho}}, u_{\chi_{\varrho}+1}, \ldots, u_{\chi_{\rho}}\right), \quad \psi=\kappa(\xi),
$$

with $\xi(t) \in \mathbb{R}^{\nu}, u(t) \in \mathbb{R}, \psi(t) \in \mathbb{R}, \chi_{0}=0, \chi_{j} \in \mathbb{R}_{>0}$ with $j=1, \ldots, \rho$, and $\phi$ and $\kappa$ smooth mappings, is a model of system (27) at $(s(\omega), l(\omega))$, i.e. system (31) has the same moment of system $(27)$ at $(s(\omega), l(\omega))$, if the equation

$$
\frac{\partial p}{\partial \omega} s(\omega)=\phi\left(p\left(\bar{\omega}_{\chi_{0}}\right), \ldots, p\left(\bar{\omega}_{\chi_{\varrho}}\right), l\left(\bar{\omega}_{\chi_{\varrho}+1}\right), \ldots, l\left(\bar{\omega}_{\chi_{\rho}}\right)\right),
$$

where $\bar{\omega}_{\chi_{i}}=\Phi_{\chi_{i}}^{s}(\omega)$, with $i=0, \ldots, \rho$, has a unique solution $p$ such that

$$
h(\pi(\omega))=\kappa(p(\omega)),
$$

where $\pi$ is the unique solution of (30). System (31) is a reduced order model of system (27) at $(s(\omega), l(\omega))$ if $\nu<n$, or if $\varrho<\varsigma$, or if $\rho<\mu$.

\section{A first family of models}

We now identify a first family of models.

Assumption 4. There exist mappings $\kappa$ and $p$ such that $\kappa(0)=$ $0, p(0)=0, p$ is locally $C^{1}$, equation (33) holds and $p$ has a local inverse $p^{-1}$.

Consistently with Definition 4, a family of models that achieves moment matching at $(s(\omega), l(\omega))$ is described by

$$
\begin{aligned}
\dot{\xi}=\Phi\left(\xi, \bar{\xi}_{\chi_{1}}, \ldots, \bar{\xi}_{\chi_{\varrho}}\right) & +\frac{\partial p(\omega)}{\partial \omega} \gamma\left(\xi_{\chi_{1}}, \ldots, \xi_{\chi_{\varrho}}\right)+ \\
& +\frac{\partial p(\omega)}{\partial \omega}\left[\sum_{j=\varrho+1}^{\rho} \delta_{j}(\xi) u_{\chi_{j}}\right], \\
\psi & =\kappa(\xi),
\end{aligned}
$$

with

$$
\begin{array}{r}
\Phi\left(\xi, \bar{\xi}_{\chi_{1}}, \ldots, \bar{\xi}_{\chi_{\varrho}}\right)=\left[\frac { \partial p ( \omega ) } { \partial \omega } \left(s(\omega)-\gamma\left(p\left(\bar{\omega}_{\chi_{1}}\right), \ldots, p\left(\bar{\omega}_{\chi_{\varrho}}\right)\right)\right.\right. \\
\left.\left.-\sum_{j=\varrho+1}^{\rho} \delta_{j}(p(\omega)) l\left(\bar{\omega}_{\chi_{j}}\right)\right)\right]_{\omega=p^{-1}(\xi)},
\end{array}
$$

where $\bar{\xi}_{\chi_{j}}=\left[\bar{\omega}_{\chi_{j}}\right]_{\omega=p^{-1}(\xi)}, k$ and $p$ are such that Assumption 4 holds, $p$ is the unique solution of (32) and $\delta$ and $\gamma$ are free mappings.

Assumption 4 holds with the selection $p(\omega)=\omega$ and $\kappa(\omega)=$ $h(\pi(\omega))$. This yields a family of models described by the equations

$$
\begin{aligned}
& \dot{\xi}=s(\xi)-\sum_{j=\varrho+1}^{\rho} \delta_{j}(\xi) l\left(\bar{\xi}_{\chi_{j}}\right)-\gamma\left(\bar{\xi}_{\chi_{1}}, \ldots, \bar{\xi}_{\chi_{\varrho}}\right)+ \\
&+\gamma\left(\xi_{\chi_{1}}, \ldots, \xi_{\chi_{\varrho}}\right)+\sum_{j=\varrho+1}^{\rho} \delta_{j}(\xi) u_{\chi_{j}}, \\
& \psi=h(\pi(\xi)),
\end{aligned}
$$

where $\delta$ and $\gamma$ are arbitrary mappings such that equation (32), namely

$$
\begin{aligned}
\frac{\partial p}{\partial \omega} s(\omega) & =s(p(\omega))-\sum_{j=\varrho+1}^{\rho} \delta_{j}(p(\omega)) l\left(p\left(\bar{\omega}_{\chi_{l}}\right)\right)- \\
& -\gamma\left(p\left(\bar{\omega}_{\chi_{1}}\right), \ldots, p\left(\bar{\omega}_{\chi_{\varrho}}\right)\right)+\gamma\left(p\left(\omega_{\chi_{1}}\right), \ldots, p\left(\omega_{\chi_{\varrho}}\right)\right)+ \\
& +\sum_{j=\varrho+1}^{\rho} \delta_{j}(p(\omega)) l\left(\omega_{\chi_{j}}\right),
\end{aligned}
$$

has the unique solution $p(\omega)=\omega$.

The nonlinear model (35) is the direct counterpart of the linear model (18). The model has several free design parameters, namely $\delta_{j}, \gamma, \chi_{j}, \varrho$ and $\rho$. We note that selecting $\gamma \equiv 0$, $\varrho=0, \rho=1$ and $\chi_{1}=0$ (in this case we define $\delta=\delta_{1}$ ), yields a reduced order model with no delays. This reduced order model coincides with the one in [17] and all results therein are directly applicable: the mapping $\delta$ can be selected to achieve matching with asymptotic stability, matching with prescribed relative degree, etc. However, as stressed previously in the paper, the choice of eliminating the delays is likely to destroy some important dynamics of the model.

Remark 6. As in the case of linear time-delay systems the results of this section can be extended to more general classes of time-delay systems provided that, for such systems, the center manifold theory applies. In particular, one can consider the class of neutral differential time-delay systems described by equations of the form

$$
\begin{aligned}
& d\left(\dot{x}_{\tau_{0}}, \ldots, \dot{x}_{\tau_{\varsigma_{1}}}\right)=f\left(x_{\tau_{\varsigma_{1}+1}}, \ldots, x_{\tau_{\varsigma_{2}}}, u_{\tau_{\varsigma_{2}+1}}, \ldots, u_{\tau_{\mu}}\right), \\
& y=h(x),
\end{aligned}
$$

with $x(t) \in \mathbb{R}^{n}, u(t) \in \mathbb{R}, y(t) \in \mathbb{R}, \tau_{0}=0, \tau_{j} \in \mathbb{R}_{>0}$ with $j=1, \ldots, \mu$ and $d, f$ and $h$ smooth mappings. The center manifold theory does not hold for this class of systems for a general mapping $d$. Specific cases have to be considered and we refer the reader to [22], [69], [66] and references therein. Note, however, that for the simple case

$$
\begin{aligned}
& \dot{x}+D \dot{x}_{\tau_{1}}=f\left(x_{\tau_{2}}, \ldots, x_{\tau_{\varsigma_{1}}}, u_{\tau_{\varsigma_{1}}+1}, \ldots, u_{\tau_{\mu}}\right), \\
& y=h(x),
\end{aligned}
$$

with $D \in \mathbb{R}^{n \times n}$, the center manifold theory holds as for standard time-delay systems if the matrix $D$ is such that $\sigma(D) \in \mathbb{D}_{<1}$.

\section{OPEN-LOOP REDUCED ORDER MODEL}

We consider now the problem of obtaining a reduced order model of an open-loop system from the closed-loop system. This problem may arise when the system to be reduced is not stable and we have to apply a feedback to use the reduction techniques proposed, yet we are interested in the reduced order model of the uncontrolled system. For ease of notation we assume that there are no delays on the input $u$.

Consider a closed-loop, nonlinear, single-input, single-output, 
continuous-time, time-delay system described by the equations

$$
\begin{aligned}
\dot{x} & =f\left(x_{\tau_{0}}, \ldots, x_{\tau_{\mu_{1}}}, u\right), \\
u & =g\left(x_{\epsilon_{0}}, \ldots, x_{\epsilon_{\mu_{2}-1}}\right)+v_{\tau_{\mu_{2}}}, \\
y & =h(x),
\end{aligned}
$$

with $x(t) \in \mathbb{R}^{n}, u(t) \in \mathbb{R}, v(t) \in \mathbb{R}, y(t) \in \mathbb{R}, \tau_{0}=0, \tau_{j} \in$ $\mathbb{R}_{>0}$ with $j=1, \ldots, \mu_{1}, \epsilon_{0}=0, \epsilon_{j} \in \mathbb{R}_{>0}$ with $j=1, \ldots, \mu_{2}$ and $f, g$ and $h$ smooth mappings. Consider a signal generator described by the equations

$$
\dot{\omega}=s(\omega), \quad \theta=l(\omega),
$$

with $\omega(t) \in \mathbb{R}^{\nu}, \theta(t) \in \mathbb{R}, s$ and $l$ smooth mappings, and the interconnected system

$$
\begin{aligned}
& \dot{\omega}=s(\omega), \\
& \dot{x}=f\left(x_{\tau_{0}}, \ldots, x_{\tau_{\mu_{1}}}, g\left(x_{\epsilon_{0}}, \ldots, x_{\epsilon_{\mu_{2}-1}}\right)+l\left(\omega_{\tau_{\mu_{2}}}\right)\right), \\
& y=h(x) .
\end{aligned}
$$

Suppose that $f(0, \ldots, 0,0)=0, g(0, \ldots, 0,0)=0, s(0)=0$, $l(0)=0$ and $h(0)=0$.

Assumption 5. There exists a unique mapping $\pi(\omega)$, locally defined in a neighborhood of $\omega=0$, which solves the partial differential equation

$$
\begin{aligned}
\frac{\partial \pi}{\partial \omega} s(\omega)=f( & \pi\left(\bar{\omega}_{\tau_{0}}\right), \ldots, \pi\left(\bar{\omega}_{\tau_{\mu}}\right), \\
& \left.g\left(\pi\left(\bar{\omega}_{\epsilon_{0}}\right), \ldots, \pi\left(\bar{\omega}_{\epsilon_{\mu_{2}-1}}\right)\right)+l\left(\bar{\omega}_{\tau_{\mu_{2}}}\right)\right),
\end{aligned}
$$

where $\bar{\omega}_{\tau_{i}}=\Phi_{\tau_{i}}^{s}(\omega)$, with $i=0, \ldots, \mu_{1}$, and $\bar{\omega}_{\epsilon_{i}}=\Phi_{\epsilon_{i}}^{s}(\omega)$, with $i=0, \ldots, \mu_{2}$.

Definition 5. Consider system (38) and the signal generator (39). Suppose Assumption 3 and 5 hold. Then the system

$$
\begin{aligned}
& \dot{\xi}=\phi\left(\xi_{\chi_{0}}, \ldots, \xi_{\chi_{\rho}}, u\right), \\
& \psi=\kappa(\xi),
\end{aligned}
$$

with $\xi(t) \in \mathbb{R}^{\nu}, u(t) \in \mathbb{R}, \chi_{0}=0, \chi_{j} \in \mathbb{R}_{>0}$, with $j=$ $1, \ldots, \rho$, and $\phi$ and $\kappa$ smooth mappings, is an open-loop model of system (38) at $(s(\omega), l(\omega))$ if the system

$$
\begin{aligned}
& \dot{\xi}=\phi\left(\xi_{\chi_{0}}, \ldots, \xi_{\chi_{\rho}}, u\right), \\
& u=g\left(\pi\left(p^{-1}\left(\xi_{\epsilon_{0}}\right)\right), \ldots, \pi\left(p^{-1}\left(\xi_{\epsilon_{\mu_{2}-1}}\right)\right)\right)+v_{\chi_{\mu_{2}}}, \\
& \psi=\kappa(\xi)
\end{aligned}
$$

with $v(t) \in \mathbb{R}, \pi$ the unique solution of (41) and $p$ invertible and the unique solution of the equation

$$
\frac{\partial p}{\partial \omega} s(\omega)=\phi\left(p\left(\bar{\omega}_{\chi_{0}}\right), \ldots, p\left(\bar{\omega}_{\chi_{\rho}}\right), l\left(\bar{\omega}_{\chi_{\mu_{2}}}\right)\right),
$$

where $\bar{\omega}_{\chi_{i}}=\Phi_{\chi_{i}}^{s}(\omega)$, with $i=0, \ldots, \rho$, such that

$$
h(\pi(\omega))=\kappa(p(\omega)),
$$

is a model of the (closed-loop) system (40) at $(s(\omega), l(\omega))$.

Obtaining a reduced order model of an open-loop system given the closed-loop system solves the problem of the reduction of nonlinear systems when their zero equilibrium is not locally exponentially stable, extending the model reduction technique by moment matching to a larger class of systems. To illustrate how to obtain an open-loop reduced order model we briefly report part of an example published in [63] without the supporting theory of this section.

Example 3. Consider the neutral model (37) of the torsional dynamics of an oilwell drillstring affected by the stick-slip phenomenon as presented in [77], [78] and [79]. The stick-slip phenomenon consists in the undesired event that a constant rotational velocity applied on the top of the string does not translate to a steady speed at the bottom of the hole. In particular the bit undergoes intervals in which it is completely blocked and intervals in which the accumulated energy is released and the rotational speed becomes larger than the prescribed value. Consider the neutral differential time-delay system described by the equations

$$
\begin{aligned}
& \dot{x}=\Upsilon \dot{x}_{\tau_{1}}-\Psi x-\Psi \Upsilon x_{\tau_{1}}- \\
& \quad-\frac{1}{I_{B}} T(x)+\frac{1}{I_{B}} \Upsilon T\left(x_{\tau_{1}}\right)+2 \frac{\Psi c_{a}}{\Lambda} \Omega_{\tau_{2}}, \\
& y=x,
\end{aligned}
$$

with

$$
\Upsilon=\frac{c_{a}-\sqrt{I G J}}{c_{a}+\sqrt{I G J}}, \quad \Psi=\frac{\sqrt{I G J}}{I_{B}}, \quad \Lambda=c_{a}-\sqrt{I G J},
$$

where $x(t)$ is the angular velocity at the bottom of the string, $y(t)$ is the output of the system, $\Omega(t)$ is the input variable, $I$ is the inertia, $J$ is the geometrical moment of inertia, $G$ is the shear modulus, $I_{B}$ is the lumped inertia representing the block at the bottom, $c_{a}$ is a constant related to the local torsion of the drillstring, $\tau_{2}=\Gamma, \tau_{1}=2 \Gamma, \Gamma=L \sqrt{\frac{I}{G J}}$ and $L$ is the length of the string. The nonlinear function $T(\cdot)$ describes the bit-rock interaction and is given by the function

$T(x)=c_{b} x+W_{o b} R_{b}\left[\mu_{c b}+\left(\mu_{s b}-\mu_{c b}\right) e^{-\frac{\gamma_{b}}{v_{f}}|x|}\right] \tanh \left(t_{g} x\right)$,

where $t_{g}$ is the gain of the hyperbolic tangent. The parameters for the simulations have been selected as in [78] and are listed in [63]. System (46), with (47), is a nonlinear neutral differential time-delay system for which the origin is not exponentially asymptotically stable and Definition 4 cannot be directly used. However, several closed-loop feedbacks have been proposed to asymptotically stabilize the origin of the system. We apply the feedback control law proposed in [78], namely

$$
\Omega(t)=k_{1} \dot{x}\left(t-\tau_{2}\right)+k_{2} x\left(t-\tau_{2}\right)+r(t) .
$$

For the closed-loop system (46), with (47) and (48), we compute numerically the solution of equation (30). A simple reduced order model achieving moment matching at $\dot{\omega}=$ $s(\omega)=0$ and belonging to the family of models (35) is described by the equations

$$
\dot{\xi}=-\delta(\xi)\left[\xi-r_{\tau_{2}}\right], \quad \psi=\pi(\xi) .
$$

We are now interested, using system (49), in obtaining a model of the open-loop system (46). By Definition 5, an open-loop 

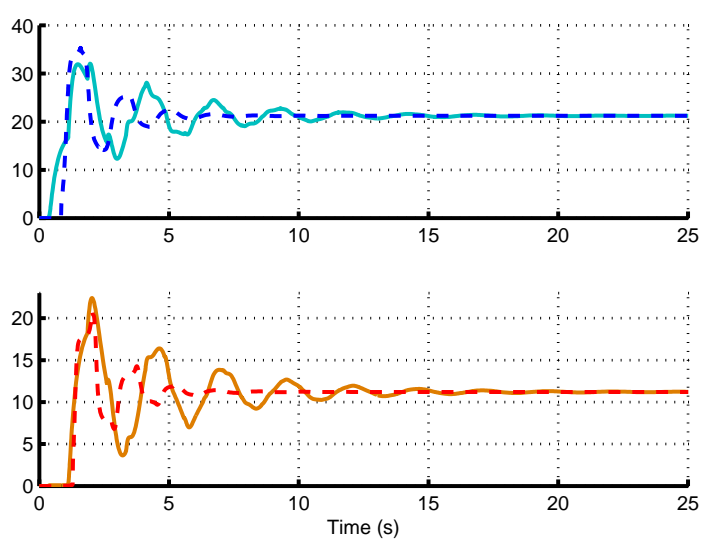

Fig. 2. Time histories of the output of system (46) (solid line) and of system (50) (dashed line) with $\delta(z)=q z^{2}+\varepsilon$ for $r=25$ (top) and $r=15$ (bottom).

model of system (46) achieving moment matching at $\dot{\omega}=0$ is given by

$$
\dot{\xi}=-\delta(\xi)\left[\xi-\mu_{\tau_{2}}\right], \quad \psi=\pi(\xi),
$$

with $\mu=-k_{1} \pi\left(\dot{\xi}_{\tau_{2}}\right)-k_{2} \pi\left(\xi_{\tau_{2}}\right)+r$.

Fig. 2 shows the time histories of the output of the openloop system (46) and of the model (50), with $\delta(z)=q z^{2}+$ $\varepsilon$, for $r=25$ (top) and $r=15$ (bottom). We can see that the model (50) and the open-loop system (46) have the same steady-state value and that, using the free mapping $\delta(\cdot)$, the transient behavior can be partially recovered.

\section{Moment AT InFInity}

We conclude the paper with the characterization of the moments at infinity for linear and nonlinear time-delay systems. The description of moment given in Definitions 1 and 3 does not characterize the moments at $s_{i}=+\infty$. Since the $k$ moments of linear delay-free systems at $s_{i}$ are defined as the first $k$ coefficients of the Laurent series expansion of the transfer function $W(s)$ at $s_{i} \in \mathbb{C}$, in a similar way the $k$ moments at infinity can be computed by evaluating the expansion at infinity of the transfer function. In addition, by using the final value theorem (see e.g. [1] and [80]), the first $k$ moments correspond to the $j=1, \ldots, k+1$ coefficients of the expansion at $t=0^{+}$of the impulse response.

Note that for differential time-delay systems, the transfer function $W(s)$ is transcendental. This implies that the computation of the limit at $s=+\infty$ has to be done with care. As noted in [80] there is widespread carelessness in the definition of the Laplace transform and its properties. In our case, it is fundamental to determine what is the meaning of the limit at infinity because the result (if well-defined) would depend upon which direction at infinity is considered. As explained in [80], in this context the limit has to be taken along the positive real axis. Then we have the following results ${ }^{5}$.

\footnotetext{
${ }^{5}$ To be coherent with equation (27) we ignore the delays in the equation of the output $y$ of the linear system (1).
}

Theorem 6. Let $\Upsilon$ be the set of values of $j=\varsigma+1, \ldots, \mu$ such that $\tau_{j}=0$.

Consider system (1).

- If $\Upsilon \neq \emptyset$ then the $k$ moments at infinity are $\eta_{k}(\infty)=$ $\sum_{j \in \Upsilon} C A_{0}^{k} B_{j}$

- If $\Upsilon=\emptyset$ then all the moments at infinity are identically zero.

Consider system (27).

- If $\Upsilon \neq \emptyset$ then the $k$ moments at infinity are $\eta_{k}(\infty)=$ $y_{I}^{(k)}\left(0^{+}\right)$, where $y_{I}^{(k)}(\cdot)$ denotes the $k$-th derivative of the impulse response of the system.

- If $\Upsilon=\emptyset$ then all the moments at infinity are identically zero.

Proof. By the equivalence between the moments at infinity and the impulse response at $t=0^{+}$, it follows that if $\tau_{j}=0$ for some $j=\varsigma+1, \ldots, \mu$ the behavior of the systems at $0^{+}$ is the same as the corresponding delay-free system (because no delay on the state has "kicked in" at $t=0^{+}$). If $\tau_{j} \neq 0$ for all $j=\varsigma+1, \ldots, \mu$ then the impulse response is delayed and it follows that the response at $0^{+}$is identically zero. Once established that the behavior is as for delay-free systems, the proof is as in [17].

Remark 7. From Theorem 6 it appears that a finite dimensional system is sufficient to characterize the moments at infinity and we can use it to match the moments at infinity as described in [17]. However, note that some properties of the transfer function are lost with a finite dimensional system. In fact if, for instance, $\tau_{j} \neq 0$ for some $j=1, \ldots, \varsigma$ and $\tau_{j}=0$ for some $j=\varsigma+1, \ldots, \mu$ the expansion at infinity along the negative real axis is identically zero, while for finite dimensional systems the expansion is the same as along the positive real axis. Or, if $\tau_{1}=\tau_{\varsigma+1}>0$ and $\tau_{j}=0$ for $i=2, \ldots, \varsigma, \varsigma+2, \ldots, \mu$, then the first coefficient of the expansion at infinity along the negative real axis is $-C B_{1}$ for time-delay systems, while zero (i.e. the same as along the positive axis) for finite dimensional systems. This suggests that a finite dimensional model that matches the moments of the system at infinity may not be a good approximation of the dynamics of the system far from $t=0$ and that to preserve the properties of the transcendental transfer function it is necessary to choose a model with the same delay structure as the original system.

\section{CONCLUSIONS}

The model reduction theory based on moment matching has been derived for linear and nonlinear differential time-delay systems. The model reduction problem has been solved first for linear time-delay systems. The description of moment has been given in terms of the unique solution of a Sylvesterlike equation. This has been subsequently extended to neutral differential time-delay systems with distributed and discrete delays. A family of systems achieving moment matching has been proposed and the problem of interpolating a larger number of points maintaining the same number of equations has been studied and solved. Then the definition of moment 
developed for linear time-delay systems has been extended to nonlinear systems by means of the center manifold theory. The moments at infinity have been characterized for both linear and nonlinear time-delay systems and a parameterized family of models achieving moment matching has been given. Finally, the solution to the problem of obtaining a reduced order model of an unstable system has been given and connections with the delay-free framework, classical results and further developments have been drawn throughout the paper.

\section{REFERENCES}

[1] A. Antoulas, Approximation of Large-Scale Dynamical Systems. Philadelphia, PA: SIAM Advances in Design and Control, 2005.

[2] V. M. Adamjan, D. Z. Arov, and M. G. Krein, "Analytic properties of Schmidt pairs for a Hankel operator and the generalized Schur-Takagi problem," Mathematics of the USSR Sbornik, vol. 15, pp. 31-73, 1971.

[3] K. Glover, "All optimal Hankel-norm approximations of linear multivariable systems and their $\mathrm{L}^{\infty}$-error bounds," International Journal of Control, vol. 39, no. 6, pp. 1115-1193, 1984.

[4] M. G. Safonov, R. Y. Chiang, and D. J. N. Limebeer, "Optimal Hankel model reduction for nonminimal systems," IEEE Transactions on Automatic Control, vol. 35, no. 4, pp. 496-502, 1990.

[5] B. C. Moore, "Principal component analysis in linear systems: Controllability, observability, and model reduction," IEEE Transactions on Automatic Control, vol. 26, no. 1, pp. 17-32, 1981.

[6] D. G. Meyer, "Fractional balanced reduction: model reduction via a fractional representation," IEEE Transactions on Automatic Control, vol. 35 , no. 12 , pp. 1341-1345, 1990.

[7] W. S. Gray and J. Mesko, "General input balancing and model reduction for linear and nonlinear systems," in European Control Conference, Brussels, Belgium, 1997.

[8] S. Lall and C. Beck, "Error bounds for balanced model reduction of linear time-varying systems," IEEE Transactions on Automatic Control, vol. 48, no. 6, pp. 946-956, 2003.

[9] H. Kimura, "Positive partial realization of covariance sequences," Modeling, Identification and Robust Control, pp. 499-513, 1986.

[10] C. I. Byrnes, A. Lindquist, S. V. Gusev, and A. S. Matveev, "A complete parameterization of all positive rational extensions of a covariance sequence," IEEE Transactions on Automatic Control, vol. 40, pp. 18411857, 1995.

[11] T. T. Georgiou, "The interpolation problem with a degree constraint," IEEE Transactions on Automatic Control, vol. 44, pp. 631-635, 1999.

[12] A. C. Antoulas, J. A. Ball, J. Kang, and J. C. Willems, "On the solution of the minimal rational interpolation problem," Linear Algebra and Its Applications, Special Issue on Matrix Problems, vol. 137-138, pp. 511573, 1990.

[13] C. I. Byrnes, A. Lindquist, and T. T. Georgiou, "A generalized entropy criterion for Nevanlinna-Pick interpolation with degree constraint," IEEE Transactions on Automatic Control, vol. 46, pp. 822-839, 2001.

[14] K. A. Gallivan, A. Vandendorpe, and P. Van Dooren, "Model reduction and the solution of Sylvester equations," in MTNS, Kyoto, 2006.

[15] C. A. Beattie and S. Gugercin, "Interpolation theory for structurepreserving model reduction," in Proceedings of the 47th IEEE Conference on Decision and Control, Cancun, Mexico, 2008.

[16] A. Astolfi, "A new look at model reduction by moment matching for linear systems," in Proceedings of the 46th IEEE Conference on Decision and Control, Dec 2007, pp. 4361-4366.

[17] — "Model reduction by moment matching for linear and nonlinear systems," IEEE Transactions on Automatic Control, vol. 55, no. 10, pp. 2321-2336, 2010.

[18] W. Dib, A. Astolfi, and R. Ortega, "Model reduction by moment matching for switched power converters," in Proceedings of the 48th IEEE Conference on Decision and Control, held jointly with the 28th Chinese Control Conference, Dec 2009, pp. 6555-6560.

[19] T. C. Ionescu and A. Astolfi, "Families of reduced order models that achieve nonlinear moment matching," in Proceedings of the 2013 American Control Conference, Washington, DC, USA, June 17-19, 2013, pp. 5518-5523.

[20] T. C. Ionescu, A. Astolfi, and P. Colaneri, "Families of moment matching based, low order approximations for linear systems," Systems \& Control Letters, vol. 64, pp. 47-56, 2014.
[21] G. Scarciotti and A. Astolfi, "Characterization of the moments of a linear system driven by explicit signal generators," in Proceedings of the 2015 American Control Conference, Chicago, IL, July, 2015.

[22] J. K. Hale, Theory of functional differential equations, ser. Applied Mathematical Sciences Series. Springer Verlag Gmbh, 1977.

[23] G. Stépán, Retarded Dynamical Systems: Stability and Characteristic Functions, ser. Pitman research notes in mathematics series. Longman Scientific \& Technical, 1989.

[24] J. K. Hale and S. M. Verduyn Lunel, Introduction to Functional Differential Equations, ser. Applied Mathematical Sciences Series. New York:Springer, 1993, vol. 99.

[25] W. Michiels and S. I. Niculescu, Stability and Stabilization of TimeDelay Systems: An Eigenvalue-Based Approach. Philadelphia: SIAM, 2007.

[26] S. I. Niculescu, Delay Effects on Stability. Heidelberg: Springer, 2001.

[27] Q. C. Zhong, Robust Control of Time-delay Systems. Germany: Springer, 2006.

[28] N. Bekiaris-Liberis and M. Krstic, Nonlinear Control Under Nonconstant Delays, ser. Advances in Design and Control. SIAM, 2013.

[29] V. Kharitonov, "Robust stability analysis of time delay systems: A survey," 4th IFAC Conference on System Structure and Control, Nantes, France, 8-10 July, vol. Penary lecture, pp. 1-12, 1998.

[30] V. B. Kolmanovskii, S. I. Niculescu, and K. Gu, "Delay effects on stability: A survey," Proceedings of the 38th IEEE Conference on Decision and Control, Phoenix, AZ, December, pp. 1993-1998, 1999.

[31] J. P. Richard, "Time-delay systems: an overview of some recent advances and open problems," Automatica, vol. 39, no. 10, pp. 1667-1694, 2003.

[32] V. B. Kolmanovskii and A. Myshkis, Introduction to the Theory and Applications of Functional Differential Equations, ser. Mathematics and Its Applications. Springer, 1999.

[33] J. K. Hale and S. M. Verduyn Lunel, "Effects of small delays on stability and control," Operator Theory: Advances and Applications, vol. 122, pp. 275-301, 2001.

[34] S. I. Niculescu, A. Trofino Nito, J. M. Dion, and L. Dugard, "Delaydependent stability of linear systems with delayed state: an LMI approach," in Proceedings of the 34th IEEE Conference on Decision and Control, vol. 2, Dec 1995, pp. 1495-1496.

[35] J. Zhang, C. R. Knospe, and P. Tsiotras, "New results for the analysis of linear systems with time-invariant delays," International Journal of Robust and Nonlinear Control, vol. 13, no. 12, pp. 1149-1175, 2003.

[36] A. W. Olbrot, "A sufficiently large time delay in feedback loop must destroy exponential stability of any decay rate," IEEE Transactions on Automatic Control, vol. 29, pp. 367-368, 1984.

[37] R. Datko, "A paradigm of ill-posedness with respect to time delays," IEEE Transactions on Automatic Control, vol. 43, no. 7, pp. 964-967, 1998.

[38] N. MacDonald, "Two delays may not destabilize although either delay can," Math Biosciences, vol. 82, pp. 127-140, 1986.

[39] J. Beddington and R. M. May, "Time lags are not necessarily destabilizing," Math. Biosciences, vol. 27, pp. 109-117, 1986.

[40] G. Abdallah, P. Dorato, J. Benitez-Read, and R. Byrne, "Delayed positive feedback can stabilize oscillatory systems," Proceedings of the 1993 American Control Conference, San Francisco, pp. 3106-3107, 1993.

[41] A. Goubet, M. Dambrine, and J. P. Richard, "An extension of stability criteria for linear and nonlinear time delay systems," IFAC Conference on System Structure and Control, Nantes, France, pp. 278-283, 1995.

[42] V. D. Blondel and A. Megretski, Unsolved Problems in Mathematical Systems and Control Theory. Princeton University Press, 2004.

[43] P. M. Mäkilä and J. R. Partington, "Laguerre and Kautz shift approximations of delay systems," International Journal of Control, vol. 72, pp. 932-946, 1999.

[44] — "Shift operator induced approximations of delay systems," SIAM Journal of Control and Optimization, vol. 37, no. 6, pp. 1897-1912, 1999.

[45] J. Zhang, C. R. Knospe, and P. Tsiotras, "Stability of linear time-delay systems: A delay-dependent criterion with a tight conservatism bound," Proceedings of the 2000 American Control Conference, Chicago, IL, June, pp. 1458-1462, 2000.

[46] S. H. Al-Amer and F. M. Al-Sunni, "Approximation of time-delay systems," Proceedings of the 2000 American Control Conference, Chicago, IL, June, pp. 2491-2495, 2000.

[47] H. T. Banks and F. Kappel, "Spline approximations for functional differential equations," Journal of Differential Equations, vol. 34, pp. 496-522, 1979

[48] G. Gu, P. P. Khargonekar, and E. B. Lee, "Approximation of infinitedimensional systems," IEEE Transactions on Automatic Control, vol. 34, no. 6, 1992. 
[49] K. Glover, J. Lam, and J. R. Partington, "Rational approximation of a class of infinite dimensional system i: Singular value of hankel operator," Mathematics of Control Circulation and Systems, vol. 3, pp. 325-344, 1990.

[50] C. Glader, G. Hognas, P. M. Mäkilä, and H. T. Toivonen, "Approximation of delay systems: A case study," International Journal of Control, vol. 53, no. 2, pp. 369-390, 1991.

[51] Y. Ohta and A. Kojima, "Formulas for Hankel singular values and vectors for a class of input delay systems," Automatica, vol. 35, pp. 201-215, 1999.

[52] M. G. Yoon and B. H. Lee, "A new approximation method for timedelay systems," IEEE Transactions on Automatic Control, vol. 42, no. 7, pp. 1008-1012, 1997.

[53] W. Michiels, E. Jarlebring, and K. Meerbergen, "Krylov-based model order reduction of time-delay systems," SIAM Journal on Matrix Analysis and Applications, vol. 32, no. 4, pp. 1399-1421, 2011.

[54] E. Jarlebring, T. Damm, and W. Michiels, "Model reduction of timedelay systems using position balancing and delay Lyapunov equations," Mathematics of Control, Signals, and Systems, vol. 25, no. 2, pp. 147$166,2013$.

[55] Q. Wang, Y. Wang, E. Y. Lam, and N. Wong, "Model order reduction for neutral systems by moment matching," Circuits, Systems, and Signal Processing, vol. 32, no. 3, pp. 1039-1063, 2013.

[56] T. C. Ionescu and O. V. Iftime, "Moment matching with prescribed poles and zeros for infinite-dimensional systems," American Control Conference, June, Montreal, Canada, pp. 1412-1417, 2012.

[57] O. V. Iftime, "Block circulant and block Toeplitz approximants of a class of spatially distributed systems-An LQR perspective,' Automatica, vol. 48, no. 12 , pp. 3098-3105, dec 2012.

[58] P. Lancaster, Theory of matrices. New York: Academic, 1969.

[59] - "Explicit solutions of linear matrix equations," SIAM Review, Vol 12, No. 4, October, 1970.

[60] Y. Halevi, "Reduced-order models with delay," International Journal of Control, vol. 64, pp. 733-744, 1996.

[61] J. Carr, Applications of Centre Manifold Theory, ser. Applied Mathematical Sciences Series. Springer-Verlag, 1981, no. v. 35.

[62] G. Scarciotti and A. Astolfi, "Model reduction by moment matching for linear time-delay systems," in 19th IFAC World Congress, Cape Town, South Africa, August 24-29, 2014, pp. 9462-9467.

[63] _ - "Model reduction by moment matching for nonlinear time-delay systems," in Proceedings of the 53rd IEEE Conference on Decision and Control, 2014, pp. 3642-3647.

[64] T. C. Ionescu and A. Astolfi, "Families of moment matching based, structure preserving approximations for linear port Hamiltonian systems," ArXiv e-prints, Apr 2013.

[65] G. Scarciotti and A. Astolfi, "Model reduction for linear systems and linear time-delay systems from input/output data," in 2015 European Control Conference, Linz, July, 2015, pp. 334-339.

[66] C. I. Byrnes, M. W. Spong, and T. J. Tarn, "A several complex variables approach to feedback stabilization of linear neutral delay-differential systems," Mathematical systems theory, vol. 17, no. 1, pp. 97-133, 1984.

[67] J. K. Hale and S. M. Verduyn Lunel, "Strong stabilization of neutral functional differential equations," IMA Journal of Mathematical Control and Information, vol. 19, no. 1-2, pp. 5-23, 2002.

[68] H. Mounier, P. Rouchon, and J. Rudolph, "Some examples of linear systems with delays," European Journal of Automatic Systems, vol. 31, no. 6, pp. 911-926, 1997.

[69] J. K. Hale, "Behavior near constant solutions of functional differential equations," Journal of differential equations, vol. 15, pp. 278-294, 1974

[70] V. B. Kolmanovskii and V. R. Nosov, Stability of Functional Differential Equations, ser. Mathematics in science and engineering. Elsevier Science, 1986

[71] R. Hotzel and M. Fliess, "On linear systems with a fractional derivation: Introductory theory and examples," Mathematics and Computers in Simulation, vol. 45, no. 3-4, pp. 385-395, 1998.

[72] R. Curtain, O. Iftime, and H. Zwart, "A comparison between LQR control for a long string of SISO systems and LQR control of the infinite spatially invariant version," Automatica, vol. 46, no. 10, pp. 1604-1615, 2010.

[73] Z. Artstein, "Linear systems with delayed controls: A reduction," IEEE Transactions on Automatic Control, vol. 27, no. 4, pp. 869-879, Aug 1982.

[74] T. Penzl, "Algorithms for model reduction of large dynamical systems," Linear Algebra and its Applications, vol. 415, no. 2-3, pp. 322 - 343, 2006, special Issue on Order Reduction of Large-Scale Systems.

[75] A. Isidori, Nonlinear Control Systems, 3rd ed., ser. Communications and Control Engineering. Springer, 1995.
[76] A. Isidori and C. I. Byrnes, "Output regulation of nonlinear systems," IEEE Transactions on Automatic Control, vol. 35, no. 2, pp. 131-140, 1990.

[77] B. Saldivar, S. Mondié, J. J. Loiseau, and V. Rasvan, "Stick-slip oscillations in oillwell drilstrings: distributed parameter and neutral type retarded model approaches," 18th IFAC World Congress, Milano, Italy, pp. 284-289, 2011.

[78] - "Exponential stability analysis of the drilling system described by a switched neutral type delay equation with nonlinear perturbations," Proceedings of the 50th IEEE Conference on Decision and Control, and European Control Conference, pp. 4164-4169, 2011.

[79] B. Saldivar and S. Mondié, "Drilling vibration reduction via attractive ellipsoid method," Journal of the Franklin Institute, vol. 350, no. 3, pp. 485-502, 2013.

[80] K. H. Lundberg, H. R. Miller, and D. L. Trumper, "Initial conditions, generalized functions, and the laplace transform - troubles at the origin," Control Systems, IEEE, vol. 27, no. 1, pp. 22-35, Feb 2007.

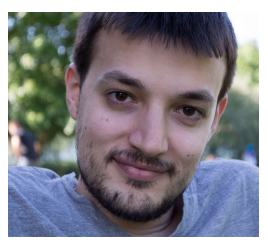

Giordano Scarciotti was born in Frascati (Rome), Italy, in 1988. He received his B.S and M.S. degree in Automation Engineering from the University of Rome Tor Vergata, Italy, in 2010 and 2012, respectively. He has been a visiting student at the Electrical and Electronic Engineering Department, Imperial College London, London, UK (2010-2011, 2012), where he is currently a Ph.D. student in the Control and Power Group. His research interests are focused on stability analysis, optimal control and model reduction.

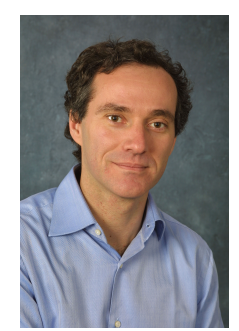

Alessandro Astolfi was born in Rome, Italy, in 1967. He graduated in electrical engineering from the University of Rome in 1991. In 1992 he joined ETH-Zurich where he obtained a M.Sc. in Information Theory in 1995 and the Ph.D. degree with Medal of Honor in 1995 with a thesis on discontinuous stabilization of nonholonomic systems. In 1996 he was awarded a Ph.D. from the University of Rome "La Sapienza" for his work on nonlinear robust control. Since 1996 he has been with the Electrical and Electronic Engineering Department of Imperial College London, London (UK), where he is currently Professor in Nonlinear Control Theory and Head of the Control and Power Group. From 1998 to 2003 he was also an Associate Professor at the Dept. of Electronics and Information of the Politecnico of Milano. Since 2005 he has also been a Professor at Dipartimento di Ingegneria Civile e Ingegneria Informatica, University of Rome Tor Vergata. He has been a visiting lecturer in "Nonlinear Control" in several universities, including ETH-Zurich (19951996); Terza University of Rome (1996); Rice University, Houston (1999); Kepler University, Linz (2000); SUPELEC, Paris (2001).

His research interests are focused on mathematical control theory and control applications, with special emphasis for the problems of discontinuous stabilization, robust and adaptive control, observer design and model reduction. He is the author of more than 120 journal papers, of 30 book chapters and of over 240 papers in refereed conference proceedings. He is the recipient of the IEEE CSS A. Ruberti Young Researcher Prize (2007) and of the IEEE CSS George S. Axelby Outstanding Paper Award (2012). $\mathrm{He}$ is a "Distinguished Member" of the IEEE CSS. He is the author (with D. Karagiannis and R. Ortega) of the monograph "Nonlinear and Adaptive Control with Applications" (Springer-Verlag).

$\mathrm{He}$ is Associate Editor of Automatica, the International Journal of Control, the Journal of the Franklin Institute, and the International Journal of Adaptive Control and Signal Processing. He is Senior Editor of the IEEE Trans. on Automatic Control and Editor-in-Chief of the European Journal of Control. $\mathrm{He}$ has also served in the IPC of various international conferences. He is currently the Chair of the IEEE CSS Conference Editorial Board. 